\title{
Article \\ Valuing the future, discounting in random environments: A review
}

\author{
Jaume Masoliver ${ }^{1,2, * \mathbb{C}}$, Miquel Montero ${ }^{1,2, * \mathbb{D}}$ and Josep Perelló ${ }^{1,2, * \mathbb{C}}$, J. Doyne Farmer ${ }^{3,4,5, * \mathbb{C}}$, John Geanakoplos ${ }^{5,6}$ \\ 1 Departament de Física de la Matèria Condensada, Universitat de Barcelona, 08028 Barcelona, Spain \\ 2 Universitat de Barcelona Institute of Complex Systems (UBICS), 08028 Barcelona, Spain \\ 3 Institute for New Economic Thinking at the Oxford Martin School \\ 4 Mathematical Institute, University of Oxford, Oxford \\ Santa Fe Institute, Santa Fe, New Mexico \\ 6 Department of Economics, Yale University, New Heaven, CT \\ * Correspondence: jaume.masoliver@ub.edu (J.M.); miquel.montero@ub.edu (M.M.); josep.perello@ub.edu \\ (J.P.); Doyne.Farmer@inet.ox.ac.uk (J.D.F); john.geanakoplos@yale.edu (J.G)
}

\begin{abstract}
We address the process of discounting in random environments which allows to value the far future in economic terms. We review several approaches to the problem regarding different well-established stochastic market dynamics in the continuous-time context and include the Feynman-Kac approach. We also review the relation between bond pricing theory and discount and introduce the market price of risk and the risk neutral measures from an intuitive point of view devoid of excessive formalism. We provide the discount for each economic model and discuss their key results. We finally present a summary of our previous empirical studies on several countries of the long-run discount problem.
\end{abstract}

Keywords: keyword 1; keyword 2; keyword 3 (List three to ten pertinent keywords specific to the article; yet reasonably common within the subject discipline.)

\section{Introduction}

The introduction around three decades ago of the view and methods of statistical physics into economics and finance signaled the appearance of a new interdisciplinary aspect of physics which is sometimes called "econophysics" [1-3]. The fact that financial prices are random with sudden and uncontrollable ups and downs has been known since very long, but the first step towards a systematic mathematical analysis of price randomness was taken by Bachelier in 1900 who proposed a model for the market dynamics in which prices follow the ordinary Brownian motion [4]. However, Bachelier's model is not completely satisfactory because in such a representation prices can be either positive or negative, contradicting one of the most fundamental principles of economics, the "principle of limited liability", which affirms that prices cannot attain negative values. This limitation in Bachelier's model was remedied more than six decades later by Osborne [5] by assuming that prices obey the geometric Brownian motion where prices are described by the exponential of the ordinary Brownian motion and, hence, they cannot ever attain negative values.

Let us denote by $S(t)$ a speculative price (or an economic index) at time $t$. In the continuous time framework, the geometric Brownian motion assumes that

$$
S(t)=S_{0} e^{x(t)},
$$

where $S_{0}=S\left(t_{0}\right)$ is the price at some initial time $t_{0}$ and $x(t)$, the so-called return, is described by the ordinary Brownian motion, that is to say, by the stochastic differential equation

$$
d x(t)=m d t+\sigma d W(t)
$$


where $W(t)$ is the standard Wiener process with zero mean and unit variance. Note that the return is assumed to be a diffusion process with constant drift $m$ and diffusion coefficient $\sigma^{2}$. In this model the return is a Gaussian process with mean and variance given respectively by $m$ and $\sigma$. The price is, hence, a log-normal process and the geometric Brownian motion is also called the log-normal model.

Despite the log-normal model is used in countless financial applications, it has some limitations [6] which have given rise to several generalizations. One of them assumes that the return is a more complex diffusion process obeying a stochastic equation of the form

$$
d x(t)=f(x, t) d t+g(x, t) d W(t),
$$

which is interpreted in the sense of Itô. In this case returns, and hence prices, are driven by an external "force" and by multiplicative noise which, in the most general case, depend explicitly of time and on the return level. Function $f(x, t)$ drives prices and function $g(x, t)$ modulates the intensity of the fluctuations around the deterministic motion set by $f(x, t)$. In any case, and regardless the values taken by $x(t)$, prices given by Eq. (1) are always nonnegative thus keeping the principle of limited liability.

Another significant shortcoming of the geometric Brownian motion model is the absence of both "fat tails" and skewness in the distribution of log-prices (i.e., returns). Indeed, empirical distributions of log-prices not only show fat tails, meaning that extreme losses and profits have a higher probability than those of the log-normal model, but also an asymmetric shape in the sense that losses are usually more probable than profits [6]. In order to address these and other problems, an intense research has aroused both in mathematics and physics which, among others, may involve the use of the Lévy process as driving noise (instead of the Wiener process), ${ }^{1}$ or models in which the variance $\sigma^{2}$ (or the noise intensity $g$ ) is a random process as in the so-called "stochastic volatility models" [9-11].

In economics and finance one of the most consequential developments is that of "discounting" which essentially tries to answer the crucial question of what is the price of the future. In other words, discounting weights the future relative to the present. Traditionally the weighting procedure has been carried through a decreasing exponential. Thus, under a constant interest rate $r$, continuously compounded, a dollar invested today at time $t=0$ yields $e^{r t}$ dollars at time $t$ [12], hence, one dollar in any future time $t$ is worth $e^{-r t}$ today. This statement is true under constant and fixed rates but in real life rates are random and this uncertainty makes completely unrealistic to represent rates by constant quantities or even by deterministic functions of time and, as a consequence, random models for rates must be addressed.

The problem of discounting is widely known in finance where it has been thoroughly studied closely related to bond pricing and specially over short periods of time [13]. The importance of discounting, particularly in the long run, does not exclusively refer to finance but to many aspects of global economy. This is, for instance, the case of long-term environmental planning which is certainly acute in climate action. Thus, in an oversimplified way, an environmental problem which costs $\mathrm{X}$ to fix at a time $t$ is worth an investment of $e^{-r t} X$ today. This analysis assumes that interest rate remains constant between today and the distant future $t$. The rate $r$ becomes a key magnitude to decide whether it becomes more beneficial to take action today with a significant investment or whether the discount gives negligible value to today's investment. The choice of discount rate is perhaps the biggest factor influencing the debate on the urgency of the response to global warming as it relates today's investments with potential climate-related losses in future [14]. No wonder that in the last years obtaining a long-run discount

\footnotetext{
1 One of the most popular alternative financial models - proposed by Mandelbrot [7] and Fama [8] in the early nineteen sixties- is provided by substituting the Wiener process by the Lévy process which takes into account the appearance of fat tails in the probability distribution of prices, a widely accepted empirical fact [6]. A major inconvenience of the Lévy process is, however, the lack of finite moments besides the first one which does not seem to be case in empirical data [6].
} 
rate valid for decades ahead has been the object of intense controversy. Thus Nicholas Stern, in an influential report commissioned by the UK government, advocates for a long-run discount of $1.4 \%$ [15] which on a 100 year horizon implies a present value of $25 \%$ (meaning the future is worth $25 \%$ as much as the present). On the other hand, Willian Nordhaus has proposed a discount rate of $4 \%$ [16] (implying a present value of $2 \%$ ) and even the higher value of $6 \%$ [17] which implies a present value of $0.3 \%$. Stern has been widely criticized for using such a low rate [18-20] and the question is far from being settled.

Economists present a variety of reasons for discounting, particularly for environmental problems in the long run. These reasons include, among others, ethical considerations [21,22], impatience, economic growth [23] and arguments based on the maximization of utility functions that are mostly chosen for mathematical convenience [24], all of them ingrained in a phenomenological expression called the Ramsey formula [25] which constitutes the standard approach to discounting in the economics literature (particularly in the long-run) [14]. From an empirical point of view, any practical economist involved in environmental debates might try to use, as the forward discount rate, the average of historical interest rates which occurred in the last 200 hundred years (2.7\% in stable countries [26]), or take the average of Wall Street forward looking models which price bonds of maturity as long as 30 years. Unfortunately, due to historical fluctuations of real interest rates, the appropriate rate is considerably below such averages [26].

In econophysics the problem of discounting, despite its relevance, is virtually unknown. The main purpose of this paper is to offer a survey of the problem devoid of excessive formalism as well as reviewing some of our recent work on the problem [26-29]

\section{The process of discounting. Fundamentals}

Let us denote by $M=M(t)$ a given quantity of wealth at time $t$. In economics the increment of $M(t)$ is assumed to be proportional to the quantity itself and the duration of the variation. For a continuous and instantaneous variation this can be written as

$$
d M(t) \propto M(t) d t
$$

This starting phenomenological law is built on the empirical observation that the bigger $M(t)$, the greater its variation at a given time along with the simpler assumption that such a variation is linear in $M(t)$ and not, for instance, quadratic.

\subsection{Definitions and general setting}

We define the interest rate as the relative time derivative

$$
r(t) \equiv \frac{1}{M(t)} \frac{d M(t)}{d t}=\frac{d \ln M(t)}{d t},
$$

i.e., the rate is the derivative of the logarithm of wealth. Let us incidentally note that linearity shown in Eq. (4) is equivalent to assume that $r(t)$ is independent of $M(t)$.

In the simplest case the law (4) represents a direct proportionality, that is to say, $r(t)$ is constant and and from (5) we see that

$$
d M(t)=r M(t) d t
$$

where $r$ is the constant interest rate which has units of $1 /$ (time) (wealth is assumed to be dimensionless). By direct integration we have the usual exponential law [12]

$$
M(t)=e^{r\left(t-t_{0}\right)} M\left(t_{0}\right),
$$


which connects wealth at some time $t_{0}$, for instance, today (which, without loss of generality, we can take equal to zero) with wealth at some future time $t>t_{0}$.

The growth law (6) appears in many branches of natural and social sciences. Thus in radioactivity if $N(t)$ represents the number of active nuclei at time $t$ the usual hypothesis is that this number decreases as

$$
d N(t)=-\lambda N(t) d t
$$

where $\lambda>0$ is the decay constant. Similar considerations apply to many other situations, as in chemical reactions or population dynamics, just to name just a few.

As we have mentioned above, discounting is the procedure of linking wealth at different times. This is done through the discount function defined as

$$
\delta(t) \equiv \frac{M(0)}{M(t)}
$$

where $M(0)$ is today's wealth. In the case of constant rates we see from Eq. (7) that this function is given by the decreasing exponential

$$
\delta(t)=e^{-r t}
$$

The assumption of constant rates is actually unrealistic. A first generalization would be to assume that rates are known functions of time $r(t)$. In such a case, the growth law (6) would be given by

$$
d M(t)=r(t) M(t) d t
$$

which after integrating yields

$$
\delta(t)=\exp \left(-\int_{0}^{t} r\left(t^{\prime}\right) d t^{\prime}\right)
$$

However, the assumption of rates being given by constants or by deterministic functions of time is unjustified, specially over long periods of time. Financial interest rates are typically described as random, as the many models for stochastic interest rates appearing in the literature show [13,30,31]. In other words, $r(t)$ is a random function of time and in consequence the discount function $\delta(t)$ given by Eq. (11) is a stochastic process. The effective discount function is then defined as the average of $\delta(t)$,

$$
D(t)=\mathbb{E}\left[\exp \left(-\int_{0}^{t} r\left(t^{\prime}\right) d t^{\prime}\right)\right],
$$

taken over all possible realization of $r(t)$. The function $r(t)$ can, in principle, be any random process. However, the most natural and simplifying assumption is that rates are Markovian processes with continuous paths, that is, rates are diffusion processes [13]. Therefore, rates are solutions to stochastic differential equations of the form

$$
d r=f(r) d t+g(r) d W(t)
$$

where $W(t)$ is the Wiener process and the equation is interpreted in the Itô sense. Note that we assume that drift $f(r)$ and noise intensity $g(r)$ do not depend explicitly on time, that is to say, the time dependence is only implicit through $r=r(t)$ which means that the interest rate process is time homogeneous and may be stationary [32]. This is certainly an idealization because real markets do not seem to be time homogeneous, at least over long periods of time [34]. 
In order to get an operative expression for the effective discount function (12) we define the additional random process

$$
x(t)=\int_{0}^{t} r\left(t^{\prime}\right) d t^{\prime}
$$

The interpretation of $x(t)$ is apparent after substituting Eq. (5) into Eq. (14) and integrating. We find

$$
x(t)=\ln \frac{M(t)}{M(0)} \quad \Rightarrow \quad M(t)=M(0) e^{x(t)},
$$

which can be taken as an alternative definition of the accumulated return $x(t)$.

Substituting Eq. (14) into Eq. (12) we see that the effective discount function can be written as

$$
D(t)=\mathbb{E}\left[e^{-x(t)}\right],
$$

which implies that, in terms of the probability density function (PDF) $p\left(x, r, t \mid r_{0}\right)$ of the bidimensional diffusion process $(x(t), r(t))$, we can write

$$
D\left(t \mid r_{0}\right)=\int_{-\infty}^{\infty} d r \int_{-\infty}^{\infty} e^{-x} p\left(x, r, t \mid r_{0}\right) d x
$$

where we have included the dependence on the initial rate, $r_{0}=r(0)$, in the discount function $D\left(t \mid r_{0}\right)$.

From Eqs. (13) and (14) we see that the bidimensional process $(x(t), r(t))$ is defined by the following pair of stochastic differential equations

$$
\begin{aligned}
d x & =r d t \\
d r & =f(r) d t+g(r) d W(t) .
\end{aligned}
$$

Therefore, the joint density obeys the (forward) Fokker-Planck equation [32]

$$
\frac{\partial p}{\partial t}=-r \frac{\partial p}{\partial x}-\frac{\partial}{\partial r}[f(r) p]+\frac{1}{2} \frac{\partial^{2}}{\partial r^{2}}\left[g^{2}(r) p\right],
$$

with the initial condition

$$
p\left(x, r, 0 \mid r_{0}\right)=\delta(x) \delta\left(r-r_{0}\right) \text {. }
$$

After solving the initial-value problem (17)-(18) and obtain the joint PDF $p\left(x, r, t \mid r_{0}\right)$, the discount function follows from Eq. (15). There are, however, two different approaches for obtaining it. One of them, which is standard in the financial literature, is based on the backward Fokker-Planck equation and it is called the Feynman-Kac approach [13]. A second procedure is based on Fourier analysis [27]. We will explain next both approaches.

\subsection{The Feynman-Kac approach}

Using this method one obtains a partial differential for the discount function $D\left(t \mid r_{0}\right)$ which is based on the backward Fokker-Planck equation for the joint density $p\left(x, r, t \mid r_{0}\right)$. In what follows we will assume that $t_{0} \neq 0$ and denote $x_{0}=x\left(t_{0}\right)$. Note that by definition $x_{0}=0$ (cf. Eq. (14)). However, we temporally keep $x_{0} \neq 0$ and set $x_{0}=0$ at the end of the calculation when needed.

The backward FPE for the PDF $p\left(x, r, t \mid x_{0}, r_{0}, t_{0}\right)$ that corresponds to the bidimensional process (16) is [32]

$$
\frac{\partial p}{\partial t_{0}}=-r_{0} \frac{\partial p}{\partial x_{0}}-f\left(r_{0}\right) \frac{\partial p}{\partial r_{0}}-\frac{1}{2} g^{2}\left(r_{0}\right) \frac{\partial^{2} p}{\partial r_{0}^{2}},
$$


with final condition as $t_{0} \rightarrow t$,

$$
p\left(x, r, t \mid x_{0}, r_{0}, t\right)=\delta\left(x-x_{0}\right) \delta\left(r-r_{0}\right)
$$

Let us observe that the problem (19)-(20) is invariant under translations of both time and $x_{0}$. We thus define the new variables

$$
t^{\prime}=t-t_{0}, \quad x^{\prime}=x-x_{0}
$$

so that

$$
\frac{\partial p}{\partial t_{0}}=-\frac{\partial p}{\partial t^{\prime}}, \quad \frac{\partial p}{\partial x_{0}}=-\frac{\partial p}{\partial x^{\prime}}
$$

and Eq. (19) reads

$$
\frac{\partial p}{\partial t^{\prime}}=-r_{0} \frac{\partial p}{\partial x^{\prime}}+f\left(r_{0}\right) \frac{\partial p}{\partial r_{0}}+\frac{1}{2} g^{2}\left(r_{0}\right) \frac{\partial^{2} p}{\partial r_{0}^{2}} .
$$

Note that under this change of variables, we also have

$$
p=p\left(x, r, t \mid x_{0}, r_{0}, t_{0}\right)=p\left(x, r, t \mid x-x^{\prime}, r_{0}, t-t^{\prime}\right)=p\left(x^{\prime}, r, t^{\prime} \mid r_{0}\right),
$$

where the last equality comes form the invariance under time and $x$ translations, that is,

$$
p\left(x, r, t \mid x_{0}, r_{0}, t_{0}\right)=p\left(x-x_{0}, r, t-t_{0} \mid r_{0}\right) .
$$

Consequently the final condition (20) becomes the initial condition

$$
p\left(x^{\prime}, r, t^{\prime}=0 \mid r_{0}\right)=\delta\left(x^{\prime}\right) \delta\left(r-r_{0}\right) .
$$

Having set the backward FPE in the form given by Eq. (22) we next obtain the equation satisfied by the effective discount $D\left(t \mid r_{0}\right)$. To this end, we multiply Eq. (22) by $e^{-x^{\prime}}$ and integrate over $x^{\prime}$ and $r$, we have

$$
\begin{aligned}
\frac{\partial}{\partial t^{\prime}} \int_{-\infty}^{\infty} d r \int_{-\infty}^{\infty} e^{-x^{\prime}} p d x^{\prime}= & -r_{0} \int_{-\infty}^{\infty} d r \int_{-\infty}^{\infty} e^{-x^{\prime}} \frac{\partial p}{\partial x^{\prime}} d x^{\prime} \\
& +\left[f\left(r_{0}\right) \frac{\partial}{\partial r_{0}}+\frac{1}{2} g^{2}\left(r_{0}\right) \frac{\partial^{2}}{\partial r_{0}^{2}}\right] \int_{-\infty}^{\infty} d r \int_{-\infty}^{\infty} e^{-x^{\prime}} p d x^{\prime}
\end{aligned}
$$

But from Eq. (15) we see that

$$
\int_{-\infty}^{\infty} d r \int_{-\infty}^{\infty} e^{-x^{\prime}} p\left(x^{\prime}, r, t^{\prime} \mid r_{0}\right) d x^{\prime}=D\left(t^{\prime} \mid r_{0}\right) .
$$

On the other hand, integrating by parts the first integral on the right hand side of Eq. (24) and using (25) we have

$$
\int_{-\infty}^{\infty} d r \int_{-\infty}^{\infty} e^{-x^{\prime}} \frac{\partial p}{\partial x^{\prime}} d x^{\prime}=\int_{-\infty}^{\infty} d r \int_{-\infty}^{\infty} e^{-x^{\prime}} p\left(x^{\prime}, r, t^{\prime} \mid r_{0}\right) d x^{\prime}=D\left(t^{\prime} \mid r_{0}\right),
$$

where we have taken into account the boundary condition (otherwise implicit in the definition of $D$ given in Eq. (15))

$$
\lim _{x^{\prime} \rightarrow \pm \infty}\left[e^{-x^{\prime}} p\left(x^{\prime}, r, t^{\prime} \mid r_{0}\right)\right]=0
$$


Substituting Eqs. (25) and (26) into Eq. (24) and setting $t_{0}=0$ which implies $t^{\prime}=t$ [cf. Eq. (21)] we finally get

$$
\frac{\partial D}{\partial t}=-r_{0} D+f\left(r_{0}\right) \frac{\partial D}{\partial r_{0}}+\frac{1}{2} g^{2}\left(r_{0}\right) \frac{\partial^{2} D}{\partial r_{0}^{2}},
$$

with the initial condition (cf. Eqs. (23) and (25))

$$
D\left(0 \mid r_{0}\right)=1
$$

The method for obtaining the discount function $D\left(t \mid r_{0}\right)$ by solving the initial-value problem (27)-(28) is called the Feynman-Kac approach and Eq. (27) the Feynman-Kac equation. In some applications (see, for instance, Sec. 3 ) it is convenient to consider $t_{0} \neq 0$ so that $t^{\prime}=t-t_{0} \neq t$. In these cases it is appropriate to denote $D=D\left(t \mid r_{0}, t_{0}\right)$ and the Feynman-Kac equation (27) reads

$$
\frac{\partial D}{\partial t_{0}}=r_{0} D-f\left(r_{0}\right) \frac{\partial D}{\partial r_{0}}-\frac{1}{2} g^{2}\left(r_{0}\right) \frac{\partial^{2} D}{\partial r_{0}^{2}},
$$

with the final condition $D\left(t \mid r_{0}, t\right)=1$.

\subsection{The Fourier transform approach.}

An alternative method for obtaining the discount function is based on the joint characteristic function., that is, on the Fourier transform of the joint density:

$$
\tilde{p}\left(\omega_{1}, \omega_{2}, t \mid r_{0}\right)=\int_{-\infty}^{\infty} e^{-i \omega_{2} r} d r \int_{-\infty}^{\infty} e^{-i \omega_{1} x} p\left(x, r, t \mid r_{0}\right) d x
$$

One of the chief advantages of working with the characteristic function is that obtaining the effective discount is straightforward. Indeed, comparison of Eq. (15),

$$
D\left(t \mid r_{0}\right)=\int_{-\infty}^{\infty} d r \int_{-\infty}^{\infty} e^{-x} p\left(x, r, t \mid r_{0}\right) d x
$$

with Eq. (30) shows that

$$
D\left(t \mid r_{0}\right)=\tilde{p}\left(\omega_{1}=-i, \omega_{2}=0, t \mid r_{0}\right)
$$

Therefore, in order to obtain the discount function we only need to know the joint characteristic function of the bidimensional process $(x, r)$. The procedure is quite advantageous in linear cases. In a forthcoming section we will apply this procedure to some standard models of interest rates.

\subsection{Adding risk aversion}

As we will see in the next section, the process of discounting just described is very closely related to an important problem in finance called bond pricing. In the context of bond pricing, there can be two kinds of investors. For one hand, if investors are risk neutral then bond prices can be modeled based on the data generating measure $p$ which is the solution of the Fokker-Planck equation (17) with initial condition (18). This is sometimes called the Local Expectation Hypothesis (LEH) [35,36]. Nonetheless, a more general assumption is that investors are sensitive to risk. In such a case bonds are somewhat more accurately priced using an artificial density $p^{*}$ usually called risk-neutral (or risk-correcting) probability measure. Both magnitudes -the data generating measure $p$ and the risk-neutral measure $p^{*}$ - are related through a quantity, denoted by $q(r, t)$ and called market price of risk which, as described in the next section, is the extra return per unit of risk that investors demand to bear risk. This additional return is thus determined by a function $q=q(r, t)$ that in its most general form may depend on the rate $r$ and 
current time $t$, although the most usual assumption is that $q=q(r)$ only depends on the rate [37]. Following a standard procedure for bond pricing [37,38] which we will present in Sect. III, one takes risk into account by replacing the drift $f(r)$ by $f^{*}(r)$,

$$
f(r) \rightarrow f^{*}(r)
$$

where

$$
f^{*}(r)=f(r)+g(r) q(r),
$$

and $q(r) \geq 0$ is the market price of risk. The form of $q(r)$ is, in principle, unknown and has to be conjectured. The simplest and most common assumption is that $q(r)=q$ is constant, in such a case the value of $q$ may be more easily estimated from empirical data. Note that now the risk-neutral measure $p^{*}\left(x, r, t \mid r_{0}\right)$ is given by the Fokker-Planck equation (17) with $f(r)$ replaced by $f^{*}(r)$, that is,

$$
\frac{\partial p^{*}}{\partial t}=-r \frac{\partial p^{*}}{\partial x}-\frac{\partial}{\partial r}\left[[f(r)+g(r) q(r)] p^{*}\right]+\frac{1}{2} \frac{\partial^{2}}{\partial r^{2}}\left[g^{2}(r) p^{*}\right],
$$

with the initial condition given by

$$
p^{*}\left(x, r, 0 \mid r_{0}\right)=\delta(x) \delta\left(r-r_{0}\right) .
$$

In an analogous way the discount function adjusted for risk will now be given by the Feynman-Kac equation (27) with $f(r)$ replaced by $f^{*}(r)$. Or, using the Fourier method, the discount function will be given in terms of the risk-neutral characteristic function, $\tilde{p}^{*}\left(\omega_{1}, \omega_{2}, t \mid r_{0}\right)$, by (cf. Eq. (31))

$$
D\left(t \mid r_{0}\right)=\tilde{p}^{*}\left(\omega_{1}=-i, \omega_{2}=0, t \mid r_{0}\right) .
$$

\section{Pricing bonds. The term structure of interest rates}

Pricing bonds is a traditional objective in finance and intimately related to the problem of discounting. It constitutes a vast subject with countless studies, many of them rather abstract, which have appeared in the mathematical finance literature during the last decades. We here present a short and intendedly simple, yet rigorous, introduction to the subject devoid as much as possible of technicalities and mathematical subtleties and refer the interested reader to more specialized works for further information [13].

A bond is a financial instrument that one purchases now and provides a payment in the future. From a more technical point of view, we say that a (discount) bond is a default-free claim on a specified sum of money to be delivered at a given future date called maturity time. Such claims are bought and issued by investors. Let us denote by $B\left(t_{0}, t\right)$ the price at time $t_{0}$ of a discount bond maturing at time $t \geq t_{0}$, with unit maturity value,

$$
B(t, t)=1 .
$$

Let us incidentally note that if the final maturity price is not 1 (say, $B(t, t)=\beta$ ) then the (initial) price of the bond would be $\beta B\left(t_{0}, t\right)$.

Bonds are classified according to the time interval to maturity $\tau$ defined as

$$
\tau=t-t_{0}
$$

Thus, if $\tau=10$ years we talk about a 10 year bond that is traded initially at $t_{0}$ (for instance, today) with price $B\left(t_{0}, t_{0}+10\right)$ and which after 10 years has unit value. Similarly for a 3 year bond, 3 month bond, etc.

The central question is to know the backward evolution of the bond price, from unit maturity to the initial purchasing price $B\left(t_{0}, t\right)$. Note that the problem is virtually identical to the problem of discounting discussed in Sect. II, with the sole difference that in discounting we look for the forward evolution from a known initial value to an 
unknown final value, while in bond pricing the situation is reverse, since we know the final value but not the initial one.

In order to proceed further we define the instantaneous rate of return $b\left(t_{0}, t\right)$ (also called forward rate) as the relative time variation of the bond price (compare with Eq. (5))

$$
b\left(t_{0}, t\right) \equiv \frac{1}{B\left(t_{0}, t\right)} \frac{\partial B\left(t_{0}, t\right)}{\partial t_{0}}=\frac{\partial \ln B\left(t_{0}, t\right)}{\partial t_{0}} .
$$

The knowledge of the forward rate $b\left(t_{0}, t\right)$ allows us to relate the initial price $B\left(t_{0}, t\right)$ and the maturing price $B(t, t)=1$. Indeed, the integration of the above equation directly leads to

$$
B\left(t_{0}, t\right)=\exp \left[-\int_{t_{0}}^{t} b\left(t_{0}^{\prime}, t\right) d t_{0}^{\prime}\right] .
$$

The close analogy between bond pricing and discounting is now apparent. Indeed the comparison of Eq. (37) with Eq. (11) shows that $B\left(t_{0}, t\right)$ is the equivalent of the discount function $\delta(t)$ and that the forward rate $b\left(t_{0}, t\right)$ is the equivalent of the discount rate $r(t)$. However, in what follows, we will use the notation $r(t)$ not for the forward rate $b\left(t_{0}, t\right)$ but for the so-called spot rate (also called nominal rate) which we will define in Eq. (39).

Another quantity of interest is the yield to maturity $y\left(t_{0}, \tau\right)$ defined by

$$
y\left(t_{0}, \tau\right) \equiv-\frac{1}{\tau} \ln B\left(t_{0}, t_{0}+\tau\right) \quad \Rightarrow \quad B\left(t_{0}, t_{0}+\tau\right)=e^{-\tau y\left(t_{0}, \tau\right)} .
$$

From (37) we see that

$$
y\left(t_{0}, \tau\right)=\frac{1}{\tau} \int_{t_{0}}^{t_{0}+\tau} b\left(t_{0}^{\prime}, t\right) d t_{0}^{\prime},
$$

that is to say, the yield is the time average of the forward rate over the maturity period $\tau$.

A final quantity is needed, the spot or nominal rate, which is defined as the limit of the yield when maturity tends to $0,{ }^{2}$

$$
r\left(t_{0}\right) \equiv \lim _{\tau \rightarrow 0} y\left(t_{0}, \tau\right)=\lim _{\tau \rightarrow 0}\left[\frac{1}{\tau} \int_{t_{0}}^{t_{0}+\tau} b\left(t_{0}^{\prime}, t\right) d t_{0}^{\prime}\right] .
$$

Solving the indeterminacy by expanding the integral in powers of $\tau$, we see that the spot rate is given in terms of the forward rate by

$$
r\left(t_{0}\right)=b\left(t_{0}, t_{0}\right) .
$$

In other words, the spot rate is the instantaneous forward rate.

Let us finally note that a loan of amount $M$ subscribed at time $t_{0}$ with an interest rate $r\left(t_{0}\right)$ (the spot rate) will, at time $t_{0}+d t_{0}$, increase in value to $M+d M$, where

$$
d M=r\left(t_{0}\right) M d t_{0} .
$$

Indeed, at any time $t_{0}$, the value of the spot rate $r\left(t_{0}\right)$ is the instantaneous increase of the loan value, that is, $r\left(t_{0}\right)=d \ln M\left(t_{0}\right) / d t_{0}$ (compare with Eq. (36)). All of this clearly heightens the close similarities with discounting mentioned above.

However, subsequent values of the spot rate are not necessarily certain. We will see next the consequences of this fact on the time evolution of the bond price $B\left(t_{0}, t\right)$.

2 In dealing with bonds one sometimes uses for the nominal rate the notation $n\left(t_{0}\right)$ instead of $r\left(t_{0}\right)$, the later reserved for real interest rates which can be negative due to inflation (see Sect. III). 


\subsection{Dynamics of the bond price}

Suppose the spot rate $r\left(t_{0}\right)$ is not deterministic but random. In such a case, and analogously to discounting, the usual assumption is that $r_{0}=r\left(t_{0}\right)$ is a Markovian random process with continuous trajectories; that is, a diffusion process obeying a stochastic differential equation of the form

$$
d r_{0}=f\left(r_{0}\right) d t_{0}+g\left(r_{0}\right) d W\left(t_{0}\right),
$$

where $W\left(t_{0}\right)$ is the standard Wiener process. We have assumed that the drift and the noise intensity are independent of time, thus the time dependence of these coefficients is implicit through $r_{0}=r\left(t_{0}\right)$. We know that this implies invariance under time translations and we can set $t_{0}=0$ when needed without loss of generality.

We will now obtain the time evolution of the bond price $B\left(t_{0}, t\right)$ from the purchasing time $t_{0}$ to maturity $t$ and to this end we follow Oldrich Vasicek [37]. Let us first observe that the most natural hypothesis consists in assuming that the bond price $B$ is a function of the initial spot rate $r_{0}=r\left(t_{0}\right)$ and write

$$
B=B\left[t_{0}, t \mid r\left(t_{0}\right)\right] .
$$

In this way $B\left(t_{0}, t \mid r_{0}\right)$ represents the price of a bond issued at time $t_{0}$ and maturing at time $t$, given that the initial interest rate is $r_{0}=r\left(t_{0}\right)$. The infinitesimal variation of the bond price is then defined by

$$
d B=B\left[t_{0}+d t_{0}, t \mid r\left(t_{0}+d t_{0}\right)\right]-B\left[t_{0}, t \mid r\left(t_{0}\right)\right] .
$$

We expand in Taylor series up to second order

$$
\begin{aligned}
B\left[t_{0}+d t_{0}, t \mid r\left(t_{0}\right.\right. & \left.\left.+d t_{0}\right)\right]=B\left[t_{0}, t \mid r\left(t_{0}\right)\right]+\frac{\partial B}{\partial t_{0}} d t_{0}+\frac{\partial B}{\partial r_{0}} d r_{0} \\
& +\frac{1}{2}\left[\frac{\partial^{2} B}{\partial t_{0}^{2}} d t_{0}^{2}+\frac{\partial^{2} B}{\partial r_{0}^{2}} d r_{0}^{2}+2 \frac{\partial^{2} B}{\partial t_{0} \partial r_{0}} d t_{0} d r_{0}\right]+\cdots
\end{aligned}
$$

Substituting for Eq. (42) and taking into account that $d W\left(t_{0}\right)=O\left(d t_{0}^{1 / 2}\right)[32,33]$ we write

$$
d B=\left[\frac{\partial B}{\partial t_{0}}+f\left(r_{0}\right) \frac{\partial B}{\partial r_{0}}\right] d t_{0}+g\left(r_{0}\right) \frac{\partial B}{\partial r_{0}} d W\left(t_{0}\right)+\frac{1}{2} g^{2}\left(r_{0}\right) \frac{\partial^{2} B}{\partial r_{0}^{2}}\left[d W\left(t_{0}\right)\right]^{2}+O\left(d t_{0}^{3 / 2}\right) .
$$

However $\left[d W\left(t_{0}\right)\right]^{2}=d t_{0}$ (in mean square sense) [32,33] and, up to first order in $d t_{0}$, we obtain

$$
d B=\left[\frac{\partial B}{\partial t_{0}}+f\left(r_{0}\right) \frac{\partial B}{\partial r_{0}}+\frac{1}{2} g^{2}\left(r_{0}\right) \frac{\partial^{2} B}{\partial r_{0}^{2}}\right] d t_{0}+g\left(r_{0}\right) \frac{\partial B}{\partial r_{0}} d W\left(t_{0}\right)
$$

Defining

$$
\mu\left(t_{0}, t \mid r_{0}\right) \equiv \frac{1}{B}\left[\frac{\partial B}{\partial t_{0}}+f\left(r_{0}\right) \frac{\partial B}{\partial r_{0}}+\frac{1}{2} g^{2}\left(r_{0}\right) \frac{\partial^{2} B}{\partial r_{0}^{2}}\right]
$$

and

$$
\sigma\left(t_{0}, t \mid r_{0}\right) \equiv-\frac{1}{B} g\left(r_{0}\right) \frac{\partial B}{\partial r_{0}}
$$

we see from (44) that the bond price satisfies the stochastic differential equation

$$
\frac{d B}{B}=\mu\left(t_{0}, t \mid r_{0}\right) d t_{0}-\sigma\left(t_{0}, t \mid r_{0}\right) d W\left(t_{0}\right),
$$


showing that the bond price is also a diffusion process.

Averaging Eq. (47) and recalling that $\mathbb{E}\left[d W\left(t_{0}\right)\right]=0$ we see that

$$
\mu\left(t_{0}, t \mid r_{0}\right)=\mathbb{E}\left[\frac{1}{B} \frac{d B}{d t_{0}}\right],
$$

which proves that $\mu\left(t_{0}, t \mid r_{0}\right)$ is the average of the instantaneous rate of return [cf. Eq. ( $36)]$ at time $t_{0}$ on a bond with maturing date $t$, given that the current spot rate is $r_{0}$. In an analogous way one can easily show that $\sigma^{2}\left(t_{0}, t \mid r_{0}\right)$ is the variance [37].

We therefore see from the above development that the bond price is a random quantity but with a final fixed price (the maturity price). The question is: what is the (initial) price that an investor has to buy (or sell) a bond at time $t_{0}$ maturing at time $t=t_{0}+\tau$ with the current spot rate $r_{0}$ ? One possible answer would be proceeding as in discounting and take the average over all possible realizations of the bond price. However, this procedure implies that the expected rate of return of a bond is invariant under risk variation -that is, under changes of the variance $\sigma^{2}\left(t_{0}, t \mid r_{0}\right)$ - a fact that investors always have in mind. We explain next a procedure resulting in a deterministic bond price which takes into account the risk aversion of investors (in practice this is only true to some extend because the mathematical procedure assumes that the market is driven by Gaussian white noise, that is, the Wiener process, which is an idealized noise presenting -among other shortcomings- no fat tails, a key characteristic of real markets [6]).

\subsection{The market price of risk}

Consider an investor who, at time $t_{0}$, sells an amount $M_{1}$ of a bond maturing at time $t_{1}$ and at the same time buys an amount $M_{2}$ of another bond with a different maturing date $t_{2}$. The total worth of the portfolio thus constructed is $M=M_{2}-M_{1}$. Note that each amount $M_{i}(i=1,2)$ is a multiple of the bond price $B\left(t_{0}, t_{i} \mid r_{0}\right)(i=1,2)$ and, hence, they also obey the stochastic differential equation (47). That is,

$$
\frac{d M_{i}}{M_{i}}=\mu\left(t_{0}, t_{i} \mid r_{0}\right) d t_{0}-\sigma\left(t_{0}, t_{i} \mid r_{0}\right) d W\left(t_{0}\right) .
$$

In consequence the infinitesimal variation $d M=d M_{2}-d M_{1}$ of the worth of the portfolio changes over time according to

$$
\begin{aligned}
d M= & {\left[\mu\left(t_{0}, t_{2} \mid r_{0}\right) M_{2}-\mu\left(t_{0}, t_{1} \mid r_{0}\right) M_{1}\right] d t_{0} } \\
& -\left[\sigma\left(t_{0}, t_{2} \mid r_{0}\right) M_{2}-\sigma\left(t_{0}, t_{1} \mid r_{0}\right) M_{1}\right] d W\left(t_{0}\right) .
\end{aligned}
$$

Suppose we choose the amounts $M_{1}$ and $M_{2}$ such that

$$
M_{1}=\frac{M}{\sigma_{1}-\sigma_{2}} \sigma_{2}, \quad M_{2}=\frac{M}{\sigma_{1}-\sigma_{2}} \sigma_{1},
$$

where $M=M_{2}-M_{1}$ and $\sigma_{i}=\sigma\left(t_{0}, t_{i} \mid r_{0}\right)(i=1,2)$. Hence $M_{1}$ is proportional to $\sigma_{2}$ while $M_{2}$ is proportional to $\sigma_{1}$. With this choice we have

$$
\sigma_{2} M_{2}-\sigma_{1} M_{1}=\sigma_{2} \frac{\sigma_{1} M}{\sigma_{1}-\sigma_{2}}-\sigma_{1} \frac{\sigma_{2} M}{\sigma_{1}-\sigma_{2}}=0,
$$

and the random term in Eq. (48) vanishes. This renders the portfolio composed of such amounts of the two bonds instantaneously riskless:

$$
d M=\frac{M}{\sigma_{1}-\sigma_{2}}\left(\mu_{2} \sigma_{1}-\mu_{1} \sigma_{2}\right) d t_{0}
$$


where $\mu_{i}=\mu\left(t_{0}, t_{i} \mid r_{0}\right)$. The rate of return $r_{M}$ of this portfolio is

$$
r_{M} \equiv \frac{1}{M} \frac{d M}{d t_{0}}=\frac{\mu_{2} \sigma_{1}-\mu_{1} \sigma_{2}}{\sigma_{1}-\sigma_{2}}
$$

In order to avoid arbitrage opportunities -that is, making profits without taking any risk- the rate $r_{M}$ must be equal to the spot rate $r_{0}$. If not, the portfolio can be purchased by taking funds borrowed at the spot rate, or otherwise sold and the profits lent out to accomplish a riskless arbitrage [37]. Therefore [compare also Eq. (41) with Eq. (50)]

$$
r_{0}=\frac{\mu_{2} \sigma_{1}-\mu_{1} \sigma_{2}}{\sigma_{1}-\sigma_{2}}
$$

Rearranging terms we get $\left(\mu_{1}-r_{0}\right) / \sigma_{1}=\left(\mu_{2}-r_{0}\right) / \sigma_{2}$, so that

$$
\frac{\mu\left(t_{0}, t_{1} \mid r_{0}\right)-r_{0}}{\sigma\left(t_{0}, t_{1} \mid r_{0}\right)}=\frac{\mu\left(t_{0}, t_{2} \mid r_{0}\right)-r_{0}}{\sigma\left(t_{0}, t_{2} \mid r_{0}\right)}
$$

This equation is valid for arbitrary maturities $t_{1}, t_{2}, \ldots$, it then follows that the ratio $\left[\mu\left(t_{0}, t \mid r_{0}\right)-r_{0}\right] / \sigma\left(t_{0}, t \mid r_{0}\right)$ must be independent of the maturity time $t$.

Let us denote by $q\left(t_{0} \mid r_{0}\right)$ the common value of such a ratio for a bond of any maturity date, given that the current spot rate (at time $t_{0}$ ) is $r_{0}$,

$$
q\left(t_{0} \mid r_{0}\right) \equiv \frac{\mu\left(t_{0}, t \mid r_{0}\right)-r_{0}}{\sigma\left(t_{0}, t \mid r_{0}\right)}, \quad\left(t \geq t_{0}\right)
$$

The quantity $q\left(t_{0} \mid r_{0}\right)$ is called the market price of risk, as it gives the variation of the expected rate of return on a bond (specified by the risk premium $\mu-r_{0}$ ) per an additional unit risk (specified by the standard deviation $\sigma$ ). ${ }^{3}$

Note that if $q=0$ the spot rate $r_{0}=r\left(t_{0}\right)$ and the average rate of return $\mu$ coincide.

$$
\mu\left(t_{0}, t \mid r_{0}\right)=r\left(t_{0}\right)
$$

$\left(t=t_{0}+\tau\right)$ meaning that the expected instantaneous rates of return on bonds are the same for all maturities.

\subsection{The term structure equation and the risk-neutral measure}

The introduction of the market price implies a non-random bond price $B=$ $B\left(t_{0}, t \mid r_{0}\right)$ which, in turn, allows a deterministic equation for the price. In effect, rewriting Eq. (51) as

$$
\mu\left(t_{0}, t \mid r_{0}\right)-r_{0}=\sigma\left(t_{0}, t \mid r_{0}\right) q\left(t_{0} \mid r_{0}\right),
$$

and substituting $\mu$ and $\sigma$ by their definitions given in Eqs. (45) and (46) we have

$$
\frac{1}{B}\left[\frac{\partial B}{\partial t_{0}}+f\left(r_{0}\right) \frac{\partial B}{\partial r_{0}}+\frac{1}{2} g^{2}\left(r_{0}\right) \frac{\partial^{2} B}{\partial r_{0}^{2}}\right]-r_{0}=-q\left(t_{0} \mid r_{0}\right) \frac{1}{B} g\left(r_{0}\right) \frac{\partial B}{\partial r_{0}}
$$

which, after rearranging terms, yields

$$
\frac{\partial B}{\partial t_{0}}=r_{0} B-\left[f\left(r_{0}\right)+g\left(r_{0}\right) q\left(t_{0} \mid r_{0}\right)\right] \frac{\partial B}{\partial r_{0}}-\frac{1}{2} g^{2}\left(r_{0}\right) \frac{\partial B}{\partial r_{0}^{2}} .
$$

This equation, called the term structure equation, is a partial differential equation for $B\left(t_{0}, t \mid r_{0}\right)$, once we know the random character of the spot rate process $r(t)$ (through its drift $f$ and noise intensity $g)$ and the market price of risk $q\left(t_{0} \mid r_{0}\right)$ is specified. Bond

\footnotetext{
3 The market price of risk $q\left(t_{0} \mid r_{0}\right)$ is the so-called Sharpe ratio [39] of the excess return $\mu-r_{0}$.
} 
prices are thus obtained after solving the deterministic equation (52) with the final condition:

$$
B\left(t, t \mid r_{0}\right)=1 .
$$

Let us observe that the term structure equation (52) for the bond price $B$ is identical to the Feynman-Kac equation (27) for the discount function $D$ as long as we make the following change of drift

$$
f\left(r_{0}\right) \longrightarrow f\left(r_{0}\right)+g\left(r_{0}\right) q\left(t_{0} \mid r_{0}\right) .
$$

On the other hand, as we have seen in Sec. 2, the solution of the Feynman-Kac equation (27) for the discount function $D\left(t \mid r_{0}\right)$ is written as the average [cf. Eq. (15)]

$$
D\left(t \mid r_{0}, t_{0}\right)=\int_{-\infty}^{\infty} d r \int_{-\infty}^{\infty} e^{-x} p\left(x, r, t \mid r_{0}, t_{0}\right) d x,
$$

where $p\left(x, r, t \mid r_{0}, t_{0}\right)$ is the probability density function of the bidimensional diffusion process $(x(t), r(t))$ defined by Eq. (16),

$$
d x=r d t, \quad d r=f(r) d t+g(r) d W(t) .
$$

Now the analogy between the term structure equation (52) and the Feynman-Kac equation (29) suggests that we can write the bond price $B\left(t_{0}, t \mid r_{0}\right)$ as an average over the different realizations of the spot rate $r\left(t_{0}\right)$. However, this averaging procedure is taken using a modified PDF called the risk-free measure. Thus, it can be proved in a more rigorous way that $[31,37]$

$$
B\left(t_{0}, t \mid r_{0}\right)=\int_{-\infty}^{\infty} d r \int_{-\infty}^{\infty} e^{-x} p^{*}\left(x, r, t \mid r_{0}, t_{0}\right) d x,
$$

where $p^{*}\left(x, r, t \mid r_{0}, t_{0}\right)$ is the risk-free measure which is the PDF of the bidimensional process $\left(x\left(t_{0}\right), r\left(t_{0}\right)\right)$ defined by the following pair of stochastic differential equations that include the market price of risk [see Eq. (54)]:

$$
\begin{aligned}
d x & =r d t \\
d r & =[f(r)+g(r) q(t \mid r)] d t+g(r) d W(t) .
\end{aligned}
$$

That is, $p^{*}$ is the solution to the FPE

$$
\frac{\partial p^{*}}{\partial t}=-r \frac{\partial p^{*}}{\partial x}-\frac{\partial}{\partial r}\left[[f(r)+g(r) q(t \mid r)] p^{*}\right]+\frac{1}{2} \frac{\partial^{2}}{\partial r^{2}}\left[g^{2}(r) p^{*}\right],
$$

with the initial condition

$$
p^{*}\left(x, r, t_{0} \mid r_{0}, t_{0}\right)=\delta(x) \delta\left(r-r_{0}\right) .
$$

Since, as we have shown in Sect. 2.2, the Feynman-Kac approach to discounting is equivalent to the Fourier method described in Sect. 2.3, we can apply the latter to obtain directly the bond price knowing only the risk neutral PDF, without having to solve the Feynman-Kac equation (52) with condition (23). Indeed, the characteristic function of the risk neutral density $p^{*}$ is the joint Fourier transform

$$
\tilde{p}^{*}\left(\omega_{1}, \omega_{2}, t \mid r_{0}, t_{0}\right)=\int_{-\infty}^{\infty} e^{-i \omega_{2} r} d r \int_{-\infty}^{\infty} e^{-i \omega_{1} x} p^{*}\left(x, r, t \mid r_{0}, t_{0}\right) d x
$$

which after comparing with Eq. (55) yields

$$
B\left(t_{0}, t \mid r_{0}\right)=\tilde{p}^{*}\left(\omega_{1}=-i, \omega_{2}=0, t \mid r_{0}, t_{0}\right) .
$$


Finally, once we know the bond price, the yield to maturity $y\left(t_{0}, \tau \mid r_{0}\right)$ (also called the term structure of interest rates) is readily evaluated from Eq. (38):

$$
y\left(t_{0}, \tau \mid r_{0}\right)=-\frac{1}{\tau} \ln B\left(t_{0}, t_{0}+\tau \mid r_{0}\right)
$$

The graphic representations of $y\left(t_{0}, \tau \mid r_{0}\right)$ as a function of $t_{0}$ and for different values of the maturity interval $\tau$ are called yield curves and are of prime importance for practitioners.

\section{Standard models}

Throughout the above development it becomes clear that in order to proceed further in the discounting process (as well as in pricing bonds) we need to identify the specific diffusion process chosen for modeling rates. Such a choice is mostly based on the analysis of empirical data $[26,28]$. Obviously in any market model proposed there are idealizations, otherwise a complete treatment of the problem would be very problematic, not to say impossible, not only analytically but computationally as well. In addition to assuming a diffusive behavior for the market, the first of such idealizations is supposing that the market is stationary, that is, the structural conditions of the market do not change in time. However, and especially after the 1980's, market circumstances have largely changed due to a great increase of transaction volumes along with transparency and, to a lesser extend, changes in investors perspectives. In this review we only address stationary models, although we are working on new models dealing with some nonstationary features of the market $[28,34]$ and we refer the interested reader to these works for further information.

On the other hand there is a property of the market that seems to be well founded on empirical grounds [6]. This is the property of mean reversion meaning that prices tend to return to some fundamental value, called normal level, which is usually identified as the long-time (i.e., stationary) mean value. The simplest way of introducing this feature in the diffusion market model is to assume a linear drift of the form $f(r)=-\alpha(r-m)$, where $\alpha>0$ is the strength of the reversion to the normal level, identified by $m$. In such a case the drift acts like a linear restoring force driving $r(t)$ towards $m$ as time increases.

\subsection{Bonds and real rates}

Before proceeding with the introduction of some standard models for the market evolution, we will briefly explain the link between bonds and (real) interest rates.

Financial economists have developed a large number of models of interest rate processes to enable them to price bonds and other cash flows. In these models interest rates are described by positive random processes since financial interest rates hardly take negative values. Although the models could be, in principle, extended to arbitrary horizons, they have only been studied carefully over time horizons of up to 30 years, since bonds are seldom issued for periods longer than this.

On the other hand, environmental economists are interested in the real behavior of the economic growth over longer horizons, in contrast to financial economists who are typically more interested in nominal rates over shorter periods of time. The behavior of real and nominal rates usually differ because due to inflation real rates can take on negative values. In this way real rates $r(t)$ are generally defined by the so-called Fisher procedure:

$$
r(t)=n(t)-i(t),
$$

where $i(t)$ is the inflation rate which is usually generated from consumer price indexes as we will explain in the next section. The quantity $n(t)$ represents nominal rates which are typically constructed out of government bonds and are usually positive (even tough in recent years, nominal rates have taken slightly negative values). In order to explain the close relationship between nominal rates and bonds, let us first recall that nominal 
rates were called spot rates in the previous section where we used there the notation $r(t)$ instead of $n(t)$. We thus define the spot (i.e., nominal) rate as (see Eqs. (39)-(40))

$$
n(t) \equiv \lim _{\tau \rightarrow 0}\left[\frac{1}{\tau} \int_{t}^{t+\tau} b\left(t^{\prime}, t\right) d t^{\prime}\right]=b(t, t),
$$

where $b\left(t^{\prime}, t\right)$ is the forward rate for bonds defined in Eq. (36) (see also Eq. (37)), that is

$$
b\left(t^{\prime}, t\right)=\frac{\partial \ln B\left(t^{\prime}, t\right)}{\partial t^{\prime}} \quad \Rightarrow \quad B\left(t^{\prime}, t\right)=\exp \left[-\int_{t^{\prime}}^{t} b\left(t^{\prime \prime}, t\right) d t^{\prime \prime}\right]
$$

where $B\left(t^{\prime}, t\right)$ is the price at time $t^{\prime}$ of a (government) bond maturing at time $t \geq t^{\prime}$. Let us recall the definition of the yield to maturity $y(t, \tau)$ given in Eq. (38),

$$
y(t, \tau) \equiv-\frac{1}{\tau} \ln B(t, t+\tau) \quad \Rightarrow \quad B(t, t+\tau)=e^{-\tau y(t, \tau)}
$$

$(\tau \geq 0)$, so that

$$
y(t, \tau)=\frac{1}{\tau} \int_{t}^{t+\tau} b\left(t^{\prime}, t\right) d t^{\prime}
$$

and comparing with Eq. (62) we see that in terms of the yield nominal rates $n(t)$ can be defined as

$$
n(t)=\lim _{\tau \rightarrow 0} y(t, \tau) .
$$

Thus for empirical analysis the yield can be used as an estimator of the nominal rates:

$$
n(t) \sim y(t, \tau)
$$

and the accuracy of such an estimator increases as $\tau \rightarrow 0$. We will return to this discussion in the next section.

In this way, taking nominal rates corrected by inflation as a proxy of economic growth, we have recently shown $[26,28,29]$ through a detailed empirical study on many countries that real interest rates are negative around $25 \%$ of the time (see next section). To understand how discounting depends on the random process used to characterize interest rates we have focussed on three different models and obtained exact analytical expressions for the discount function [27]. The three models describe to varying degree a number of relevant characteristics observed in data, while being simple enough to allow for complete analytical treatment. Their main results are summarized in Table 1.

The first model is based on the Ornstein-Uhlenbeck (OU) process -also called Vasicek model in the financial literature [13]- which allows for negative rates and is, therefore, suitable for pricing environmental problems. The model has a stationary probability distribution and exhibits reversion to the mean, which means that the process tends to return to its average stationary value. We will review this model below.

The second and third models we have considered are given by the Feller and log-normal processes respectively. For these processes rates cannot be negative. The Feller process -also known as the Cox-Ingersoll-Ross (CIR) model [41]- has reversion to the mean and stationary probability distribution. It is one of the most popular models in finance [13] and we have recently review the main properties of the Feller process in previous works $[27,40]$. A third model, also implying positive rates, is the lognormal process (occasionally called Dotham model in the financial literature [43]). The model does not have reversion to the mean nor a stationary distribution. Despite these shortcomings the log-normal process has also been used in the financial literature mainly because is positive and allows for analytical treatment [13]. We refer the interested reader to our previous work [27] for details on this model.

As remarked in the introduction we are primarily interested in valuing the far future for environmental problems rather than the short time discount of finance, the latter 
implying positive interest rates while the former involving positive as well as negative rates. For this reason we next review with more detail the Vasicek model allowing for both positive and negative rates than the CIR and log-normal models of which we only present a sketched review.

Table 1. Key statistical features for three standard models: the Vasicek (Ornstein-Uhlenbeck), the Cox-Ingersoll-Ross (Feller), and the Log-normal models. Average and variance are provided in terms of the model parameters to better compare the long run discount $D(t)$ asymptotic behavior. The long run discount is provided showing an exponential decay with long-run rate of discount $r_{\infty}$ for the Vasicek and the Cox-Ingersoll-Ross models, jointly with an specific combination of parameters in the Log-normal case ( $k^{2} / 2<\alpha$, mild fluctuations). $\delta$ is defined in Eq. (113).

\begin{tabular}{llllll}
\hline \hline Model & $\mathbb{E}[r(t)]$ & $\operatorname{Var}[r(t)]$ & $D(t \rightarrow \infty)$ & & $r_{\infty}$ \\
\hline Vasicek & $m$ & $k^{2} / \alpha$ & $\exp \left(-r_{\infty} t\right)$ & & $m-k^{2} / 2 \alpha^{2}$ \\
Feller & $m$ & $m k^{2} /(2 \alpha)$ & $\exp \left(-r_{\infty} t\right)$ & & $\frac{2 m}{1+\sqrt{1+2 k^{2} / \alpha}}$ \\
Log-normal & $r_{0} e^{\alpha t}$ & $r_{0}^{2} e^{2 \alpha t}\left[e^{k^{2} t}-1\right]$ & constant & $\left(k^{2} / 2>\alpha\right)$ & -- \\
& & & $\exp \left(-r_{\infty} t\right)$ & $\left(k^{2} / 2<\alpha\right)$ & $\left(\alpha-k^{2} / 2\right) / \delta$ \\
& & $t^{-1 / 2}$ & $\left(k^{2} / 2=\alpha\right)$ & -- \\
\hline \hline
\end{tabular}

\subsection{The Vasicek (Ornstein-Uhlenbeck) model}

In this model, rates are described by the Ornstein-Uhlenbeck process [37], that is a diffusion model with linear drift and constant noise intensity:

$$
d r(t)=-\alpha[r(t)-m]+k d W(t)
$$

where $r(t)$ is the rate and $W(t)$ the Wiener process. The parameter $m$ (the normal level) is a mean value to which rates revert, $k>0$ is the amplitude of fluctuations, and $\alpha>0$ is the strength of the reversion to the mean. These parameters have to be estimated from empirical data.

In this case the Fokker-Planck equation for the joint density $p\left(x, r, t \mid r_{0}\right)$ of the bidimensional process $(x(t), r(t))$ defined in (16) reads (cf. (17)). Therefore, the joint density obeys the Fokker-Planck equation

$$
\frac{\partial p}{\partial t}=-r \frac{\partial p}{\partial x}+\alpha \frac{\partial}{\partial r}[(r-m) p]+\frac{1}{2} k^{2} \frac{\partial^{2} p}{\partial r^{2}}
$$

with the initial condition

$$
p\left(x, r, 0 \mid r_{0}\right)=\delta(x) \delta\left(r-r_{0}\right) .
$$

The joint Fourier transform of these equations results in a simpler initial-value problem for the joint characteristic function $\tilde{p}\left(\omega_{1}, \omega_{2}, t \mid r_{0}\right)$,

$$
\frac{\partial \tilde{p}}{\partial t}=\left(\omega_{1}-\alpha \omega_{2}\right) \frac{\partial \tilde{p}}{\partial \omega_{2}}-\left(i m \omega_{2}+\frac{1}{2} k^{2} \omega_{2}^{2}\right) \tilde{p},
$$

and

$$
\tilde{p}\left(\omega_{1}, \omega_{2}, 0 \mid r_{0}\right)=e^{-i \omega_{2} r_{0}} .
$$

We seek the solution to this problem in the form of a Gaussian function:

$$
\tilde{p}\left(\omega_{1}, \omega_{2}, t \mid r_{0}\right)=\exp \left\{-A\left(\omega_{1}, t\right) \omega_{2}^{2}-B\left(\omega_{1}, t \mid r_{0}\right) \omega_{2}-C\left(\omega_{1}, t \mid r_{0}\right)\right\}
$$

where $A\left(\omega_{1}, t\right), B\left(\omega_{1}, t\right)$, and $C\left(\omega_{1}, t\right)$ are unknown functions to be consistently determined. Substituting the ansatz (70) into Eq. (68), identifying like powers in $\omega_{2}$ and 
taking into account Eq. (69), we find that these functions satisfy the following set of differential equations

$$
\begin{array}{ll}
\dot{A}=-2 \alpha A-k^{2} / 2, & A\left(\omega_{1}, 0\right)=0 ; \\
\dot{B}=-\alpha B+2 \omega_{1} A-i m \alpha, & B\left(\omega_{1}, 0\right)=i r_{0} ; \\
\dot{C}=\omega_{1} B, & C\left(\omega_{1}, 0\right)=0 .
\end{array}
$$

These equations are a set of linear ordinary differential equations which can be sequentially integrated, with the result

$$
\begin{gathered}
A\left(\omega_{1}, t\right)=\frac{k^{2}}{4 \alpha}\left(1-e^{-2 \alpha t}\right), \\
B\left(\omega_{1}, t \mid r_{0}\right)=i r_{0} e^{-\alpha t}+\frac{k^{2} \omega_{1}}{2 \alpha^{2}}\left(1-2 e^{-\alpha t}+e^{-2 \alpha t}\right)+i m\left(1-e^{-\alpha t}\right),
\end{gathered}
$$

and

$$
\begin{aligned}
C\left(\omega_{1}, t \mid r_{0}\right)= & i \omega_{1} r_{0} \frac{1}{\alpha}\left(1-e^{-\alpha t}\right)+\frac{k^{2} \omega_{1}^{2}}{2 \alpha^{3}}\left[\alpha t-2\left(1-e^{-\alpha t}\right)+\frac{1}{2}\left(1-e^{-2 \alpha t}\right)\right] \\
& +i m \omega_{1}\left[t-\frac{1}{\alpha}\left(1-e^{-\alpha t}\right)\right] .
\end{aligned}
$$

We have thus proved that the joint characteristic function $\tilde{p}\left(\omega_{1}, \omega_{2}, t \mid r_{0}\right)$ is given by the Gaussian function (70) with the coefficients given above. Note that in terms of the bidimensional distribution (70) the distribution of the rate is given by the marginal PDF

$$
p\left(r, t \mid r_{0}\right)=\int_{-\infty}^{\infty} p\left(x, r, t \mid r_{0}\right) d x
$$

and the characteristic function of the rate then reads

$$
\tilde{p}\left(\omega_{2}, t \mid r_{0}\right)=\tilde{p}\left(\omega_{1}=0, \omega_{2}, t \mid r_{0}\right)
$$

This results in the Gaussian density

$$
\tilde{p}\left(\omega_{2}, t \mid r_{0}\right)=\exp \left\{-\frac{k^{2}}{4 \alpha}\left(1-e^{-2 \alpha t}\right) \omega_{2}^{2}-i\left[r_{0} e^{-\alpha t}+m\left(1-e^{-\alpha t}\right)\right] \omega_{2}\right\} .
$$

In the stationary state $(t \rightarrow \infty)$ we have

$$
\tilde{p}_{s t}\left(\omega_{2}\right)=e^{-\left(k^{2} / 4 \alpha\right) \omega_{2}^{2}-i m \omega_{2}} \quad \Longrightarrow \quad p_{s t}(r)=\left(\frac{\alpha}{\pi k^{2}}\right)^{1 / 2} e^{-\alpha(r-m)^{2} / k^{2}},
$$

which proves that the normal level $m$ is the stationary mean value,

$$
m=\mathbb{E}[r(t)]
$$

It can also be shown that the correlation function of the process, defined as the average

$$
C(\tau)=\mathbb{E}[r(t+\tau) r(t)]-[\mathbb{E}[r(t)]]^{2},
$$

$(\tau \geq 0)$ in the stationary state reads [27]

$$
C(\tau)=\left(k^{2} / 2 \alpha\right) e^{-\alpha \tau},
$$


which means that $\alpha^{-1}$ is the correlation time, $\tau_{c}$, of the rate. Indeed

$$
\tau_{c} \equiv \frac{1}{C(0)} \int_{0}^{\infty} C(\tau) d \tau=\alpha^{-1} .
$$

Let us observe that the volatility, $\sigma^{2}=C(0)$, is independent of the normal level and given by

$$
\sigma^{2}=k^{2} / 2 \alpha \text {. }
$$

The discount function $D\left(t \mid r_{0}\right)$ is also obtained from Eqs. (70) - (73) although in this case after setting $\omega_{1}=-i$ and $\omega_{2}=0$ (cf. Eq. (31)). We have

$$
\begin{aligned}
\ln D(t) & =-\frac{r_{0}}{\alpha}\left(1-e^{-\alpha t}\right) \\
& +\frac{k^{2}}{2 \alpha^{3}}\left[\alpha t-2\left(1-e^{-\alpha t}\right)+\frac{1}{2}\left(1-e^{-2 \alpha t}\right)\right]-m\left[t-\frac{1}{\alpha}\left(1-e^{-\alpha t}\right)\right],
\end{aligned}
$$

which after rearranging terms can be written as

$$
\ln D(t)=-\left(m-\frac{k^{2}}{2 \alpha^{2}}\right) t+\frac{1}{\alpha}\left[m-r_{0}-\frac{k^{2}}{4 \alpha^{2}}\left(3-e^{-\alpha t}\right)\right]\left(1-e^{-\alpha t}\right),
$$

where $r_{0}=r(0)$ is the initial rate. Note that as $t \rightarrow \infty$ (in fact when $t \gg \alpha^{-1}$, i.e., for times much grater than the correlation time $\alpha^{-1}$ ) Eq. (79) shows at once that the discount function of the Vasicek model has the typical exponential decay

$$
D(t) \simeq e^{-r_{\infty} t}
$$

where

$$
r_{\infty}=m-k^{2} / 2 \alpha^{2},
$$

is the long-run discount rate. Let us note that the long-run rate can be defined as the limit

$$
r_{\infty}=-\lim _{t \rightarrow \infty} \frac{\ln D(t)}{t},
$$

as long as the limit exists. Let us also note the important fact that $r_{\infty}$ is smaller than the mean value of the return given by the normal level $m$. This reduction is quantified by the "noise-to-signal" ratio $k / \alpha$, which means that either a long persistence (recall that this is equivalent to long correlation time, i.e., $\alpha$ small) or an increase of the noise fluctuations (i.e., $k$ large) reduce the long-run discount rate as compared with the average rate.

We finally easily see from Eq. (79) that as $t \rightarrow 0$ the discount function approximates to $D(t) \simeq e^{-r_{0} t}$ which would correspond to a fixed interest rate without random fluctuations or deterministic changes.

\section{Risk aversion}

As mentioned above risk aversion is taken into account by introducing the market price of risk $q(r)$ and changing drift according to Eq. (32). For the Vasicek model, in which $f(r)=-\alpha(r-m)$ and $g(r)=k$, we have

$$
f^{*}(r)=-\alpha(r-m)+k q(r),
$$

and assuming $q(r)=q$ to be a constant independent of $r$, we write

$$
f^{*}(r)=-\alpha\left(r-m^{*}\right),
$$

where

$$
m^{*}=m+\frac{q k}{\alpha} .
$$


Since the modified drift $f^{*}(r)$ has the same form that $f(r)$ we conclude that the adjustedfor-risk discount function will be given by Eq. (79) after the replacement $m \rightarrow m^{*}$. In particular, the adjusted long-run discount now reads [cf. Eq. (81)]

$$
r_{\infty}^{*}=m+\frac{q k}{\alpha}-\frac{k^{2}}{2 \alpha^{2}} .
$$

We thus see that the long-run discount depends on the historical rate $m$, but this is shifted by two terms. The first term raises the long-run rate due to the market price of risk. The second shift lowers it by an amount given by the ratio of uncertainty (as measured by $k$ ) and persistence (as measured by $\alpha$ ). We rewrite Eq. (86) as

$$
r_{\infty}^{*}=m+\frac{k}{\alpha}\left(q-\frac{k}{2 \alpha}\right) .
$$

This makes it clear that whether or not the overall shift in the long-run discount rate is positive or negative depends on the size of the market price of risk and on the noise-tosignal ratio between the volatility parameter and the reversion rate.

It is not surprising that the market price of risk raises the long term rate, but it is not so obvious that uncertainty and persistence can lower it. Indeed for the OrsteinUhlenbeck process it can make it arbitrarily small. For any given mean interest rate $m$, by varying $k$ and $\alpha$, the long-run discount rate $r_{\infty}$ can take on any value less than $m$, including negative values, while at the same time the standard deviation $\sigma$ can also be made to take on any arbitrary positive value.

A negative long-run rate is due to the amplification of negative real interest rates $r(t)$. Computation of the discount function involves an average over exponentials, rather than the exponential of an average. As a result, periods where interest rates are negative are amplified, and can easily dominate periods where interest rates are large and positive, even if the negative rates are rarer and weaker. It does not take many such periods to substantially reduce the long run interest rate.

To summarize, in the Vasicek model, and even taking into account risk aversion, the long-run discounting rate can be much lower than the mean, and indeed can correspond to low interest rates that are rarely observed.

\subsection{The Cox-Ingersoll-Ross (Feller) model}

In the financial literature one of the most accepted models for interest rates is the Cox-Ingersoll-Ross (CIR) model [41] where rates follow the Feller process described by drift and noise intensity given respectively by [42]

$$
f(r)=-\alpha(r-m), \quad g(r)=k \sqrt{r} .
$$

The Feller model is thus a diffusion process described by the stochastic differential equation

$$
d r(t)=-\alpha[r(t)-m] d t+k \sqrt{r(t)} d W(t),
$$

where $W(t)$ is the standard Wiener process and, as in the OU process, $m>0$ represents the mean stationary rate (the normal level) and $\alpha^{-1}$ is the correlation time [27]. Let us note that since the diffusion coefficient in one-dimensional diffusions is given by the square of the noise intensity, we see that the Feller process has a linear diffusion vanishing at the origin. This turns the origin into a singular boundary which results in significant properties for the process [40].

As in the Vasicek model, the linear drift results in a restoring force which, in the absence of noise, makes the process decay toward the normal level $m$. On the other hand, the state-dependent noise intensity $k \sqrt{r}$ for large values of $r$ magnifies the effect of noise while when $r$ goes to zero this effect vanishes. Therefore, as the process approaches the origin the drift drags $r$ towards $m$. Hence, since $m>0$, starting at some positive value 
$r_{0}>0$ the process cannot attain negative values, with the overall result that the Feller process remains always positive.

We have reviewed rather thoroughly the properties of the Feller process and refer the reader to [27] and [40] for a more detailed information.

The process is not Gaussian and the stationary PDF as $t \rightarrow \infty$ is the Gamma distribution [27]

$$
p_{s t}(r)=\frac{\left(2 \alpha / k^{2}\right)^{\theta}}{\Gamma(\theta)} r^{\theta-1} e^{-\left(2 \alpha / k^{2}\right) r},
$$

where

$$
\theta=\frac{2 \alpha m}{k^{2}}
$$

is a positive and dimensionless constant which combines all the parameters of the model into a single expression. As mentioned above a major characteristic of the Feller process is that $r(t)$ cannot attain negative values which makes the model a convenient tool for pricing bonds which are never negative [13].

In the Feller model the joint density of the discounting process $(x(t), r(t))$ defined in Eq. (16) obeys the Fokker-Planck equation (FPE) (cf. Eqs. (17)-(18))

$$
\frac{\partial p}{\partial t}=-r \frac{\partial p}{\partial x}+\alpha \frac{\partial}{\partial r}[(r-m) p]+\frac{k^{2}}{2} \frac{\partial^{2}}{\partial r^{2}}(r p),
$$

with the initial condition

$$
p\left(x, r, 0 \mid r_{0}\right)=\delta(x) \delta\left(r-r_{0}\right) .
$$

The joint Fourier transform, Eq. (30), turns Eqs. (92)-(93) into a more manageable problem:

$$
\begin{gathered}
\frac{\partial \tilde{p}}{\partial t}=\left(\omega_{1}-\alpha \omega_{2}-i \frac{k^{2}}{2} \omega_{2}^{2}\right) \frac{\partial \tilde{p}}{\partial \tilde{\omega}_{2}}-i \alpha m \omega_{2} \tilde{p}, \\
\tilde{p}\left(\omega_{1}, \omega_{2}, 0 \mid r_{0}\right)=e^{-i \omega_{2} r_{0}} .
\end{gathered}
$$

Equation (94) is a linear partial differential equation of first order whose solution can be obtained by the method of characteristics and we refer the interested reader to our work [27] for a detailed information. Once we know the solution $\tilde{p}\left(\omega_{1}, \omega_{2}, t \mid r_{0}\right)$, the discount function is then obtained through Eq. (31) with the result [27]

$$
D(t)=\left[\frac{2 \lambda e^{-(\lambda-\alpha) t / 2}}{(\lambda+\alpha)+(\lambda-\alpha) e^{-\lambda t}}\right]^{\theta} \exp \left\{-\frac{2\left(1-e^{-\lambda t}\right) r_{0}}{(\lambda+\alpha)+(\lambda-\alpha) e^{-\lambda t}}\right\},
$$

where $\theta$ is defined in Eq. (91) and

$$
\lambda=\sqrt{\alpha^{2}+2 k^{2}} .
$$

Notice that $\lambda>\alpha$ and the time scale represented by $\lambda^{-1}$ is smaller than the correlation time $\alpha^{-1}$.

In this case the long-run discount rate, defined by the limit (cf. Eq. (82))

$$
r_{\infty}=-\lim _{t \rightarrow \infty} \frac{\ln D(t)}{t}
$$

is directly obtained from Eq. (96) with the result

$$
r_{\infty}=\frac{1}{2}(\lambda-\alpha) \theta,
$$


and, as in the Vasicek model, the effective discount reduces to the expected exponential decay

$$
D(t) \simeq e^{-r_{\infty} t} \quad(t \rightarrow \infty) .
$$

Substituting into Eq. (98) the expressions for $\theta$ and $\lambda$ given in Eqs. (91) and (97) we write

$$
r_{\infty}=\frac{2 m}{1+\sqrt{1+2 k^{2} / \alpha^{2}}}
$$

which clearly shows that the long-run discount rate is always smaller than the stationary average rate:

$$
r_{\infty}<m
$$

Figure 1 shows the discount function $D(t)$ along with the quantity $-\ln D(t) / t$ (cf. Eq. (82)) and compare them with the Vasicek model with equivalent parameters.

Risk aversion

For the Feller process, the adjusted drift for risk defined in Eq. (54), reads

$$
f^{*}(r)=-\alpha(r-m)+k q(r) \sqrt{r}
$$

where $q(r)$ is the market price of risk as discussed in the previous section. For any function $q(r)$ (including a constant market price of risk $q$ ) this adjusted drift leads to an unsolvable Fokker-Planck equation with no analytical expression for the adjusted discount and the long-run discount rate. It is, nonetheless, possible to get analytical expressions for these quantities if the market price of risk has the following functional form

$$
q(r)=q \sqrt{r},
$$

where $q \geq 0$ is a positive quantity. In such a case we may write

$$
f^{*}(r)=-\alpha^{*}\left(r-m^{*}\right)
$$

where

$$
\alpha^{*}=\alpha-k q, \quad m^{*}=\frac{\alpha m}{\alpha-k q} .
$$

The adjusted drift has the same form than $f(r)$. Therefore, the adjusted discount function will be given Eq. (96) with the replacements $\alpha \rightarrow \alpha^{*}$ and $m \rightarrow m^{*}$ and the long-run discount is [cf. Eq. (100)]

$$
r_{\infty}^{*}=\frac{2 m^{*}}{1+\sqrt{1+2 k^{2} / \alpha^{* 2}}}
$$

From the definitions of $\alpha^{*}$ and $m^{*}$ we easily see that $\alpha^{*} \leq \alpha$ and $\alpha^{*} m^{*}=\alpha m$. Hence, writing $r^{*}$ as

$$
r_{\infty}^{*}=\frac{2 \alpha^{*} m^{*}}{\alpha^{*}+\sqrt{\alpha^{* 2}+2 k^{2}}} \geq \frac{2 \alpha m}{\alpha+\sqrt{\alpha^{2}+2 k^{2}}}=r_{\infty},
$$

so that $r_{\infty}^{*} \geq r_{\infty}$ and if the market price of risk has the form given in Eq. (102) then in the CIR model risk always increases the long-run discount rate regardless noise intensity and persistence.

\subsection{The log-normal model}

In this model rates are described by the the geometric Brownian motion (log-normal process) and the model is determined by the stochastic differential equation

$$
\frac{d r}{r}=\alpha d t+k d W(t)
$$


where $r$ is the interest rate, $\alpha$ and $k$ are constant parameters, $\alpha$ may be positive or negative whereas $k$ is always positive and $W(t)$ is the standard Wiener process. Equation (106) can be integrated at once yielding

$$
r(t)=r_{0} \exp \left\{\left(\alpha-\frac{k^{2}}{2}\right) t+k W(t)\right\}
$$

showing that $r(t)$ is never negative $\left(r_{0}>0\right)$. Therefore, the log-normal model is more suited for modeling nominal interest rates and bonds in finance than for the long-run real rates of environmental economics. Contrary to $\mathrm{OU}$ and Feller processes, the log-normal process does not show reversion to the mean. Indeed, as $t$ increases we see from Eq. ( 107) that the rate either diverges when $\alpha>0$ or goes to zero if $\alpha<0$. In an equivalent way one can also show from Eq. (107) that the mean and variance of the process are [27]

$$
\langle r(t)\rangle=r_{0} e^{\alpha t}, \quad \operatorname{Var}[r(t)]=r_{0}^{2} e^{2 \alpha t}\left(e^{k^{2} t}-1\right) .
$$

The discount associated with the log-normal process model was studied in 1978 by L. U. Dothan [43] and in finance it is sometime refereed to as the Dothan model. Because it allows for analytical treatment it is one of the models used in the literature [13]. For this model the FPE for the joint density of the discounting processes $(x(t), r(t))$ is given by (cf. Eq. (17))

$$
\frac{\partial p}{\partial t}=-r \frac{\partial p}{\partial x}-\alpha \frac{\partial}{\partial r}(r p)+\frac{1}{2} k^{2} \frac{\partial^{2}}{\partial r^{2}}\left(r^{2} p\right),
$$

with the usual initial condition given by Eq. (18). The Fourier transform of this expression leads to the following equation for the characteristic function $\tilde{p}\left(\omega_{1}, \omega_{2}, t \mid r_{0}\right)$

$$
\frac{\partial \tilde{p}}{\partial t}=\left(\omega_{1}+\alpha \omega_{2}\right) \frac{\partial \tilde{p}}{\partial \tilde{\omega}_{2}}+\frac{1}{2} k^{2} \omega_{2}^{2} \frac{\partial^{2} \tilde{p}}{\partial \tilde{\omega}_{2}^{2}}
$$

and the initial condition (93). Eq. (108) is a partial differential equation of second order which cannot be solved by the method of characteristics and we refer the interested reader to our work [27] for a detailed information on how to solve Eq. (109) using the time-Laplace transform. Hence -and contrary to Vasicek and CIR models where it is possible to obtain exact expressions for the discount function $D(t)$ - for the log-normal case we can only achieve the exact expression of its Laplace transform,

$$
\hat{D}(s)=\int_{0}^{\infty} e^{-s t} D(t) d t
$$

The resulting formula -written as an integral of special functions, the Kummer functionis rather intricate and we will not write it here (see [27] for more information). However, from the exact expression for $\hat{D}(s)$ we can get asymptotic expressions as $t \rightarrow \infty$ of the discount function $D(t)$ in real time. This is done using the so-called Tauberian theorems which relate the small $s$ behavior of $\hat{D}(s)$ with the long-time behavior of $D(t)[44,45]$. The final result is the following asymptotic expression for the discount function $D(t)$ in the long run as $t \rightarrow \infty$ [27]

$$
D(t) \sim \begin{cases}\text { constant } & k^{2} / 2>\alpha, \\ e^{-r_{\infty} t} & k^{2} / 2<\alpha, \\ t^{-1 / 2} & k^{2} / 2=\alpha .\end{cases}
$$

The asymptotic form of the discount function thus depends on the values taken by the ratio $\alpha / k^{2}$ between the strength of the constant deterministic drift $\alpha$ and the amplitude of fluctuations given by $k^{2} / 2$ (which can be considered the "signal-to-noise ratio" of this model). 
(i) The case $k^{2} / 2>\alpha$ corresponds to strong fluctuations, where the noise intensity $k^{2} / 2$ is greater than the drift parameter $\alpha$. In this case the discount tends to a constant value (for the actual value of this constant see [27]).

(ii) The case $k^{2} / 2<\alpha$ corresponds to mild fluctuations for which the deterministic drift is stronger than noise. In such a case the discount function has the expected exponential decay

$$
D(t) \sim e^{-r_{\infty} t}
$$

with a long-run rate of discount given by [27]

$$
r_{\infty}=\frac{1}{\delta}\left(\alpha-\frac{k^{2}}{2}\right)
$$

where $\delta>1$ is a positive numerical factor which only depends on the ratio $2 \alpha / k^{2}$ and reads

$$
\delta=\psi\left(2 \alpha / k^{2}\right)+\frac{1}{2 \alpha / k^{2}-1},
$$

where $\psi(\cdot)$ is the digamma function.

Let us write Eq. (111) in a more characteristic form. Indeed, from Eq. (107) we see that

$$
\mathbb{E}\left[\ln \frac{r(t)}{r_{0}}\right]=\left(\alpha-\frac{k^{2}}{2}\right) t
$$

and with the help of Eq. (111) we write Eq. (111) as

$$
D(t) \sim \exp \left\{-\frac{1}{\delta} \mathbb{E}\left[\ln \frac{r(t)}{r_{0}}\right]\right\},
$$

$\left(t \rightarrow \infty\right.$ and $\left.k^{2} / 2<\alpha\right)$. Note that the average $\mathbb{E}\left[\ln r(t) / r_{0}\right]$ is what a practitioner would take as an estimate of the discount rate up to time $t$ within the log-normal model. Since $\delta>1$, the analytical result (114) shows that the actual long-run rate of the model is a fraction of the average rate. We have shown elsewhere that the long-run discount rate is at most $73 \%$ of the average rate [26]. In this way when $2 \alpha / k^{2}>1$ the log-normal model follows a similar pattern to that of the OU and Feller models: In all of them the long-run rate is smaller than the average rate. This general statement is in fact a direct consequence of Jenesen's inequality, which states that the average of a convex function is greater than or equal to the function of the average; that is, $\mathbb{E}[f(X)] \geq f(\mathbb{E}[X])$. Assuming $f$ to be the decreasing exponential and $X$ the cumulative process $x(t)$ defined in Eq. (14), it follows immediately that the long-run rate $r_{\infty}$ must be always less than or equal to the average rate. Nonetheless, our procedure quantifies the difference among averages [27].

(iii) The critical case $\alpha=k^{2} / 2$, in which deterministic motion and fluctuations are balanced, leads to the hyperbolic discount function as obtained by Farmer and Geanakoplos $[46,47]$. The hyperbolic $D(t)$ is substantially greater than any exponential decaying function, showing that there is no long-run rate of interest in this case. In fact the long-run rate of interest is 0 , but that does not convey as precise information as saying $D(t)$ is approximately $k / \sqrt{t}$ for all large $t$. Since the sum (i.e., the integral) of all these $D(t)$ is infinite, such $D(t)$ assign infinite value to any permanent positive flow of consumption: the infinite future is infinitely valuable.

\section{Risk aversion}

Let us very briefly comment on the inclusion of risk aversion in the Dothan model. For the log-normal process $f(r)=\alpha r$ and $g(r)=k r$ and

$$
f^{*}(r)=[\alpha+k q(r)] r
$$




\begin{tabular}{|c|c|c|c|c|c|c|}
\hline & Country & Consumer Price Index & Bond Yields & from & to & records \\
\hline 1 & Italy & $\begin{array}{l}\text { CPITAM } \\
\text { annual from } 12 / 31 / 1861 \\
\text { quarterly from } 12 / 31 / 1919\end{array}$ & $\begin{array}{l}\text { IGITA10 } \\
\text { quarterly }\end{array}$ & $12 / 31 / 1861$ & $09 / 30 / 2012$ & 565 \\
\hline 2 & Chile & $\begin{array}{l}\text { CPCHLM } \\
\text { quarterly }\end{array}$ & $\begin{array}{l}\text { IDCHLM } \\
\text { quarterly }\end{array}$ & $03 / 31 / 1925$ & $09 / 30 / 2012$ & 312 \\
\hline 3 & Canada & $\begin{array}{l}\text { CPCANM } \\
\text { quarterly }\end{array}$ & $\begin{array}{l}\text { IGCAN10 } \\
\text { quarterly }\end{array}$ & $12 / 31 / 1913$ & $09 / 30 / 2012$ & 357 \\
\hline 4 & Germany & $\begin{array}{l}\text { CPDEUM } \\
\text { annual from } 12 / 31 / 1820 \\
\text { quarterly from } 12 / 31 / 1869\end{array}$ & $\begin{array}{l}\text { IGDEU10 } \\
\text { quarterly }\end{array}$ & $12 / 31 / 1820$ & $09 / 30 / 2012$ & 729 \\
\hline 5 & Spain & $\begin{array}{l}\text { CPESPM } \\
\text { annual from } 12 / 31 / 1821 \\
\text { quarterly from } 12 / 31 / 1920\end{array}$ & $\begin{array}{l}\text { IGESP10 } \\
\text { quarterly }\end{array}$ & $12 / 31 / 1821$ & $09 / 30 / 2012$ & 709 \\
\hline 6 & Argentina & $\begin{array}{l}\text { CPARGM } \\
\text { annual from } 12 / 31 / 1864 \\
\text { quarterly from } 12 / 31 / 1932\end{array}$ & $\begin{array}{l}\text { IGARGM } \\
\text { quarterly }\end{array}$ & $12 / 31 / 1864$ & $03 / 31 / 1960$ & 342 \\
\hline 7 & Netherlands & $\begin{array}{l}\text { CPNLDM } \\
\text { annual }\end{array}$ & $\begin{array}{l}\text { IGNLD10D } \\
\text { annual }\end{array}$ & $12 / 31 / 1813$ & $12 / 31 / 2012$ & 189 \\
\hline 8 & Japan & $\begin{array}{l}\text { CPJPNM } \\
\text { quarterly }\end{array}$ & $\begin{array}{l}\text { IGJPN10D } \\
\text { quarterly }\end{array}$ & $12 / 31 / 1921$ & $12 / 31 / 2012$ & 325 \\
\hline 9 & Australia & $\begin{array}{l}\text { CPAUSM } \\
\text { annual from 12/31/1861 } \\
\text { quarterly 12/31/1991 }\end{array}$ & $\begin{array}{l}\text { IGAUS10 } \\
\text { quarterly }\end{array}$ & $12 / 31 / 1861$ & $09 / 30 / 2012$ & 564 \\
\hline 10 & Denmark & $\begin{array}{l}\text { CPDNKM } \\
\text { annual from 12/31/1821 } \\
\text { quarterly from 12/31/1914 }\end{array}$ & $\begin{array}{l}\text { IGDNK10 } \\
\text { quarterly }\end{array}$ & $12 / 31 / 1821$ & $09 / 30 / 2012$ & 725 \\
\hline 11 & South Africa & $\begin{array}{l}\text { CPZAFM } \\
\text { quarterly }\end{array}$ & $\begin{array}{l}\text { IGZAF10 } \\
\text { quarterly }\end{array}$ & $12 / 31 / 1920$ & $09 / 30 / 2012$ & 329 \\
\hline 12 & Sweden & $\begin{array}{l}\text { CPSWEM } \\
\text { annual }\end{array}$ & $\begin{array}{l}\text { IGSWE10 } \\
\text { annual }\end{array}$ & $12 / 31 / 1868$ & $09 / 30 / 2012$ & 135 \\
\hline 13 & United Kingdom & $\begin{array}{l}\text { CPGBRM } \\
\text { annual }\end{array}$ & $\begin{array}{l}\text { IDGBRD* } \\
\text { annual }\end{array}$ & $12 / 31 / 1694$ & $12 / 31 / 2012$ & 309 \\
\hline 14 & United States & $\begin{array}{l}\text { CPUSAM } \\
\text { annual }\end{array}$ & $\begin{array}{l}\text { TRUSG10M } \\
\text { annual }\end{array}$ & $12 / 31 / 1820$ & $10 / 30 / 2012$ & 183 \\
\hline
\end{tabular}

Table 1: List of the data analyzed. Notes (i) Chile: we have taken the Discount (ID) rate since the Government Bond Yield data was not available. (ii) Germany: From 06/30/1915 to $03 / 31 / 1916$ IGDEU is empty and the previous available record has been repeated. (iii) Spain: From $07 / 31 / 1936$ to $12 / 31 / 1940$ no records available. $07 / 31 / 1936$ is empty and the previous available record has been repeated . (iv) Netherlands: $2 / 31 / 1945$ is empty and the previous available record has been repeated. (v) Japan: From 12/31/1946 to $09 / 30 / 1948$ is empty and the previous available record has been repeated.

Assuming a constant market price of risk, $q(r)=q \geq 0$, we have

$$
f^{*}(r)=\alpha^{*} r, \quad \alpha^{*}=\alpha+q .
$$

Again $f^{*}(r)$ has the same form than $f(r)$ and all previous results will apply after making the replacement $\alpha \rightarrow \alpha+q$.

\section{Some empirical results}

In order to choose an appropriate model for rates, which would allow us to obtain realistic long-run discount functions, we performed a rather complete empirical study on interest rates combined with inflation. Our study follows the line partly initiated by Newell and Pizer [48] (see also [49]). To our knowledge there are few empirical studies on real rates, with some exceptions. We remark here the recent and excellent survey by Giglio, Maggiori and Stroebel on the housing market in London and Singapore [50,51] which has allowed for a rather realistic estimation of long-run discount rates.

Our first concern was knowing how the discount process depended on the underlying random process that characterizes interest rates. To this end we collected data for nominal interest rates and inflation of fourteen countries over time spans ranging from 
87 to 318 years [26]. The countries in our sample are: Argentina (ARG, 1864-1960), Australia (AUS, 1861-2012), Chile (CHL, 1925-2012), Germany (DEU, 1820-2012), Denmark (DNK, 1821- 2012), Spain (ESP, 1821-2012), United Kingdom (GBR, 1694-2012), Italy (ITA, 1861-2012), Japan (JPN, 1921-2012) (see Table I), Netherlands (NLD, 1813-2012), Sweden (SWE, 1868-2012), the United States (USA, 1820-2012), and South Africa (ZAF, 1920-2012). Data is reported in Table 1.

Since all but two of our nominal interest rate processes are for ten year government bonds, which pay out over a ten year period, we smoothed out inflation rates with a ten year moving average, and subtracted the annualized inflation index from the annualized nominal rate to compute the real interest rate, as explained in the previous section by means of the Fisher's procedure (cf. Eq, (61)),

$$
r(t)=n(t)-i(t),
$$

where $n(t)$ is the nominal rate and $i(t)$ the inflation rate. The particular case of United States is plotted in Figure 2.

In our empirical analysis nominal rates are determined by IG rates constructed from the 10 year Government Bond Yield $y(t, \tau)$ with $\tau=10$ years. Thus, looking at Eqs. (63) and (64) we estimate nominal rates by

$$
n(t) \sim y(t, \tau=10 \text { years }) .
$$

Let us recall that denoting by $B(t, t+\tau)$ the government bond issued at time $t$ and maturing at time $t+\tau$ with unit maturity, $B(t, t)=1$, the yield $y(t, \tau)$ is defined as (cf. Eq. (38))

$$
y(t, \tau) \equiv-\frac{1}{\tau} \ln B(t, t+\tau) \quad \Longrightarrow \quad B(t, t+\tau)=e^{-\tau y(t \mid \tau)} .
$$

One can argue that $\tau=10$ years is not a short period of time in order to consider $y(t, \tau=10$ years) a very accurate estimator of $n(t)$ (cf. Eqs. (63) and (64)). Although this may be true we must bear in mind that 10 years bonds are the shortest bonds available for most of the countries analyzed.

The inflation rate is estimated through the Consumer Price Index (CPI) as

$$
i(t) \sim \frac{1}{\tau} \ln [I(t+\tau) / I(t)],
$$

where $I(t)$ is the aggregated inflation up to time $t$, and $\tau=10$ years. The relation between $I(t)$ and the Consumer Price Index (CPI) is

$$
I(t+\tau)=I(t) \prod_{j=0}^{\tau-1}[1+C(t+j)]
$$

where $C(t)$ is the time series of the empirical CPI. The instantaneous rate of inflation $i(t)$ is, therefore, estimated by the quantity $i(t+\tau)$ which written in terms of the CPI reads

$$
i(t) \sim i(t+\tau)=\frac{1}{\tau} \sum_{j=0}^{\tau-1} \ln [1+C(t+j)] .
$$

A remarkable characteristic observed in many epochs for all countries is that real interest rates frequently become negative, often by substantial amounts and over long periods of time as shown in Table 2 (see also Fig. 2). On average, real interest rates are negative one quarter of the time. This makes the Feller and log-normal models -as well as any other model assuming positive interest rates [13]- less interesting for addressing real interest rates and environmental problems. We have, therefore, confined 


\begin{tabular}{lcrrrrrrrr}
\hline \hline Country & Neg RI & $\hat{m}$ & Min & Max & $\hat{k}$ & Min & Max & $\hat{\alpha}$ & $\hat{r}_{\infty}$ \\
\hline Italy & $28 \%(40 y)$ & -0.3 & -9.1 & 5.6 & 6.9 & 0.8 & 10.1 & 0.22 & -5.4 \\
Chile & $56 \%(43 y)$ & -6.8 & -20.2 & 12.0 & 25.2 & 5.6 & 44.1 & 0.40 & -26 \\
Canada & $22 \%(20 y)$ & $\mathbf{2 . 9}$ & 0.1 & 6 & $\mathbf{2 . 3}$ & 1.1 & 2.0 & 0.26 & $\mathbf{2 . 5}$ \\
Germany & $14 \%(25 y)$ & -10.7 & -51.0 & 4.0 & 33.9 & 0.9 & 61.4 & 0.20 & -160 \\
Spain & $25 \%(45 y)$ & 5.7 & -0.5 & 13.5 & 2.9 & 1.2 & 3.6 & 0.06 & -6.4 \\
Argentina & $20 \%(17 y)$ & $\mathbf{2 . 4}$ & -2.9 & 6.8 & $\mathbf{6 . 2}$ & 2.8 & 6.7 & 0.39 & $\mathbf{1 . 1}$ \\
Netherlands & $17 \%(33 y)$ & $\mathbf{3 . 2}$ & 0.8 & 5.4 & $\mathbf{1 . 6}$ & 0.8 & 2.2 & 0.14 & $\mathbf{2 . 4}$ \\
Japan & $33 \%(26 y)$ & -2.2 & -7.8 & 4.0 & 9.7 & 1.1 & 13.2 & 0.24 & -10 \\
Australia & $23 \%(33 y)$ & $\mathbf{2 . 6}$ & -0.7 & 4.9 & $\mathbf{2 . 3}$ & 0.7 & 2.8 & 0.19 & $\mathbf{1 . 9}$ \\
Denmark & $18 \%(33 y)$ & $\mathbf{3 . 2}$ & 1.5 & 4.3 & $\mathbf{2 . 3}$ & 1.1 & 2.9 & 0.23 & $\mathbf{2 . 7}$ \\
South Africa & $43 \%(36 y)$ & $\mathbf{1 . 8}$ & -2.2 & 5.5 & $\mathbf{2 . 5}$ & 1.2 & 2.0 & 0.21 & $\mathbf{1 . 1}$ \\
Sweden & $28 \%(38 y)$ & $\mathbf{2 . 3}$ & -0.3 & 3.9 & $\mathbf{2 . 5}$ & 0.6 & 3.4 & 0.25 & $\mathbf{1 . 9}$ \\
United Kingdom & $14 \%(45 y)$ & $\mathbf{3 . 3}$ & 1.4 & 4.3 & $\mathbf{1 . 9}$ & 1.0 & 2.4 & 0.19 & $\mathbf{2 . 8}$ \\
United States & $31 \%(36 y)$ & $\mathbf{2 . 6}$ & 1.0 & 4.0 & $\mathbf{1 . 8}$ & 1.2 & 2.1 & 0.18 & $\mathbf{2 . 1}$ \\
\hline Stable countries & $23 \%(33 y)$ & $\mathbf{2 . 7}$ & -0.14 & 5.0 & $\mathbf{2 . 6}$ & 1.04 & 2.94 & 0.23 & $\mathbf{2 . 1}$ \\
Unstable counntries & $31 \%(36 y)$ & $-\mathbf{2 . 9}$ & 17.7 & 1.8 & $\mathbf{1 6}$ & 1.9 & 26.5 & 0.22 & $-\mathbf{4 2}$ \\
\hline \hline
\end{tabular}

Table 2: Parameter estimation of the OU (Vasicek) model (using real rates) in yearly units using stationary averages. Notes: (i) "Neg RI" gives the percentage of time and the total number of years in which real interest rates are negative. (ii) The columns $\hat{m}, \hat{k}$ (in \%) and $\hat{\alpha}$ are estimates taking each country time series; $\hat{r}_{\infty}$ (in \%) is evaluated from Eq. (81). (iii) The Min and Max columns illustrate the robustness of the estimation procedure by providing the minimum and the maximum value of parameter estimation on four data blocks of equal length. (iv) The parameter $\alpha$ is estimated by fitting the empirical correlation function to an exponential (cf. Eq. (77)) after using the whole data block. (v) Countries in boldface are those considered historically more stable with positive long-run rates $\hat{r}_{\infty}>0$.

the empirical work to the OU (Vasicek) model. We also assume the Local Expectation Hypothesis for which we live in a risk neutral world and the market price of risk is zero $[35,36,38]$.

We can estimate the parameters $m, \alpha$ and $k$ of the Vasicek model to each of the data series. There are several feasible procedures. One possible way is to deal with stationary averages. The parameter $m$ is easily estimated because it is the stationary mean value of the rate [cf. Eq. (76)]

$$
m=\mathbb{E}[r(t)] .
$$

The estimation of parameters $\alpha$ and $k$ is based on the correlation function of the OrnsteinUhlenbeck process. Thus from Eq. (77) we have

$$
C\left(t-t^{\prime}\right)=\frac{k^{2}}{2 \alpha} e^{-\alpha\left|t-t^{\prime}\right|}
$$

Evaluating then the empirical correlation from data and fitting it by an exponential we estimate $\alpha$ (measured in 1 /year units) for each country. The third and last parameter, $k$, is obtained from the empirical standard deviation $\sigma^{2}=\mathbb{E}\left[|r(t)-m|^{2}\right]$, which for the Vasicek model is given by Eq. (78). That is,

$$
k=\sigma \sqrt{2 \alpha}
$$

The resulting parameters for all countries are listed in Table 2 along with its maximum and minimum value for each country. This procedure allows us to show that parameters may indeed fluctuate on different periods of time. 
Once the parameters of the Vasicek model have been estimated, the long-run discount rate is readily evaluated from Eq. (81),

$$
r_{\infty}=m-k^{2} / 2 \alpha^{2} .
$$

For this calculation, we have neglected market price of risk as mentioned above.

The fourteen countries divide into two very clear groups. Nine countries, with relative stable (that is, positive) real interest rates, have long-run positive rates (indicated in boldface type in Table 2). The average historical rate for these nine countries is $\bar{m}=2.7 \%$ while the average long-run rate is $\bar{r}_{\infty}=2.1 \%$ which, on average, is $29 \%$ lower than $\bar{m}$. Five countries with less stable behavior have long-run negative rates and an exponentially increasing discount. We can term these countries as unstable and it may not be a coincidence that all five have experienced fascist governments. Four cases of this group have a negative average rate $m$ due to at least one period of runaway inflation; the exception is Spain, which has a (highly positive) mean real interest rate, but still has a long-run negative rate. In every case convergence to the long-run rate happens within 30 years, and typically within less than a decade. This is in contrast to other treatments of fluctuating rates, which assume short term rates are always (or nearly always) positive and predict that the decrease in the discounting rate happens over a much longer timescale, which can be measured in hundreds years $[48,49,52-55]$.

Alternatively, we can estimate parameters using the well-established maximum likelihood procedure. Maximum likelihood estimation for the Vasicek model is extensively documented in the financial mathematics literature (see for instance [13]). The approach differs from the previous one as it focuses its attention on two consecutive steps of our time series (generally consecutive years) and takes the conditional probability to perform the estimation. Table 3 shows that the most inaccurate estimator is $\hat{\alpha}$, a not surprising fact since the estimation of $\alpha$ is known to be difficult for the Vasicek model [56]. The last two columns in Table 3 include the long-run interest rate estimator $\hat{r}_{\infty}$ and its error calculated through error propagation.

Only four countries (Netherlands, Sweden, United Kingdom and United States) show a positive long-run rate, $r_{\infty}>0$. This estimation procedure leads to more to more negative $r_{\infty}$. This feature can be attributed to the fact that in most of the countries estimating $\alpha$ via maximum likelihood brings smaller values which in turn leads to more drastic corrections to the long-run rate as $r_{\infty}$ is inversely proportional to $\alpha$ (remind that $\left.r_{\infty}=m-k^{2} / 2 \alpha^{2}\right)$. This effect is particularly relevant in most turbulent countries during last century (e.g. Germany) thus signaling a more intense lack of stationarity in empirical data. The averaged $r_{\infty}$ over all countries estimated via maximum likelihood is also sensitively smaller but if we focus the attention on stable countries (with $r_{\infty}>0$ ) both estimation procedures bring quite similar results (see for instance United States case in Tables 2 and 3, 2.1\% versus $1.8 \%$ ). As in the previous estimation procedure, we have also neglected the effects of risk aversion and the market price of risk.

As mentioned above the Vasicek model is the only one among the three most classic models allowing for negative rates. This is the reason why we have excluded both the Feller and the lognormal models from our analysis. Let us nonetheless briefly study what modifications should be carried out in order to use these positive rate models in our analysis. For the Cox-Ingersoll-Ross (Feller) model it is possible to redefine the model by shifting the process $y=r-r_{\min }$ where $r_{\min }<0$. The estimation through maximum likelihood procedure and its error analysis is then possible [56] and Figure 1 provides the shifted Cox-Ingersoll-Ross discount curve when taking data from United States and compares it with the equivalent result assuming the Vasicek model. In Ref. [28] we show with more detail how to redefine the Feller process and how estimation could be possible. 


\begin{tabular}{lrrrrrrrr}
\hline \hline Country & $\hat{m}$ & $\sigma_{\hat{m}}$ & $\hat{\alpha}$ & $\sigma_{\hat{\alpha}}$ & $\hat{k}^{2}$ & $\sigma_{\hat{k}^{2}}$ & $\hat{r}_{\infty}$ & $\sigma_{\hat{r}_{\infty}}$ \\
\hline Italy & 1.97 & 15.95 & 0.0056 & 0.0089 & 0.1146 & 0.068 & -177.8 & 19.2 \\
Chile & -5.79 & 31.46 & 0.0201 & 0.0227 & 31.07 & 2.49 & -391.7 & 44.2 \\
Canada & 2.66 & 3.91 & 0.0142 & 0.0178 & 0.275 & 0.021 & -4.15 & 3.94 \\
Germany & -9.45 & 66.95 & 0.0071 & 0.0089 & 41.72 & 2.19 & -4094 & 228 \\
Spain & 6.71 & 6.92 & 0.0167 & 0.0137 & 2.371 & 0.126 & -35.78 & 7.28 \\
Argentina & 3.15 & 7.09 & 0.0228 & 0.0231 & 2.240 & 0.171 & -18.31 & 7.27 \\
Netherlands & $\mathbf{5 . 9 9}$ & 0.78 & 0.1648 & 0.0550 & $\mathbf{1 . 7 9 7}$ & 0.243 & $\mathbf{5 . 6 6}$ & 0.78 \\
Japan & 5.02 & 24.68 & 0.0053 & 0.0114 & 1.396 & 0.109 & -243.1 & 31.4 \\
Australia & 3.97 & 4.50 & 0.0089 & 0.0112 & 0.223 & 0.013 & -10.29 & 4.58 \\
South Africa & 2.69 & 4.72 & 0.0154 & 0.0193 & 0.435 & 0.034 & -6.49 & 4.77 \\
Sweden & $\mathbf{2 . 7 9}$ & 1.66 & 0.0676 & 0.0317 & 1.692 & 0.206 & $\mathbf{0 . 9 5}$ & 1.67 \\
Denmark & 4.10 & 2.59 & 0.0161 & 0.0133 & 0.315 & 0.017 & -1.97 & 2.61 \\
United Kingdom & $\mathbf{3 . 4 2}$ & 0.62 & 0.1635 & 0.0326 & 3.137 & 0.253 & $\mathbf{2 . 8 3}$ & 0.62 \\
United States & $\mathbf{3 . 1 9}$ & 1.23 & 0.0603 & 0.0257 & $\mathbf{1 . 0 0 3}$ & 0.105 & $\mathbf{1 . 8 1}$ & 1.24 \\
\hline Stable countries & $\mathbf{3 . 8 5}$ & 1.07 & 0.1140 & 0.0362 & $\mathbf{1 . 9 0 7}$ & 0.202 & $\mathbf{2 . 8 1}$ & 1.08 \\
Unstable countries & 1.50 & 16.86 & 0.0132 & 0.0150 & 8.120 & 0.523 & -498.4 & 35.3 \\
\hline \hline
\end{tabular}

Table 3: Maximum likelihood estimation for the Vasicek model and the long-run interest rate. $\hat{m}$ is the estimator of the mean real interest rate in 1 /years (in \%). $\hat{\alpha}$ is the estimator related to the characteristic reversion time in 1 /year. The squared root of the estimator of $k^{2}$ is the volatility of the process and $k^{2}$ is given in terms of $1 /$ (year) ${ }^{3}$ (multiplied by $10^{4}$ to be comparable with results in Table 2$)$. These estimators are accompanied with the square root of the variance, $\sigma^{\prime}$ s, of each estimator. $\hat{r}_{\infty}$ is the subsequent estimator of the long-run real interest rate in 1/year (in \%). Negative values of $\hat{r}_{\infty}$ mean the discount function is asymptotically increasing and its standard error is obtained through error propagation. The last two rows show separately the average over all countries, the stable countries with $r_{\infty}>0$ and the unstable countries with $r_{\infty}<0$. In all three rows standard error provided corresponds to the standard deviation of the $\hat{r}_{\infty}$ for the different countries. 


\section{Discussion}

We have reviewed one of the most important aspects of economics and finance as is the problem of discount which weights the future relative to the present. The problem is obviously very relevant in finance over relatively short time spans, but it is even more crucial for long-run planning in addressing environmental problems on how to act now with measures to mitigate the effects of climate change. To our knowledge this is a rather unknown issue to the econophysics community and this review is specially intended to them. We have thus addressed the problem with the simplest possible approach and yet with a high level of rigor and generality. In this way we have also developed the traditional method used in mathematical finance to address the problem as is the Feynman-Kac approach. In addition we have reviewed the bond pricing theory and its close similarity with discounting and presented a short introduction to the term structure of interest rates along with the market price of risk.

We obtain quantitative results on the problem by studying with some detail three standard models for the dynamical evolution of rates. These models are based on the Ornstein-Uhlenbeck process (the Vasicek model), allowing for both positive and negative rates, and also on the Feller and log-normal processes for positive rates. We have presented exact results for the discount function and asymptotic expressions as $t \rightarrow \infty$ leading to the long-run discount rate and discuss the modifications of these expressions when the market price of risk is taken into account. An important conclusion is that for all models the long-run discount rate is always less than the long-time average rate. A conclusion which necessarily has to have consequences in any long-run economic planning.

We have finally reviewed our recent empirical study on 14 different countries which has allowed to obtain numerical values of the parameters which appear in the Vasicek model. We have shown two different estimation procedures and briefly discuss the differences and similarities.

Author Contributions: Conceptualization, J.M., M.M., J.P., J.D.F. and J.G.; methodology, J.M., M.M., J.P., J.D.F. and J.G.; formal analysis, J.M., M.M., J.P., J.D.F. and J.G.; investigation, J.M., M.M., J.P., J.D.F. and J.G.; resources, J.M., M.M., J.P., J.D.F. and J.G.; writing-original draft preparation, J.M.; writing—review and editing,J.M., M.M., J.P., J.D.F. and J.G.; funding acquisition, M.M., and J.P. All authors have read and agreed to the published version of the manuscript.

Funding: This research was funded by MINEICO (Spain), Agencia Estatal de Investigación (AEI) grant number PID2019-106811GB-C33 (AEI/10.13039/501100011033) (JM, MM, JP); by Generalitat de Catalunya grant number 2017 SGR 608 (JM, MM, JP); by National Science Foundation grant 0624351 (JG); and by the Institute for New Economic Thinking (JDF).

Conflicts of Interest: 'The authors declare no conflict of interest. The funders had no role in the design of the study; in the collection, analyses, or interpretation of data; in the writing of the manuscript, or in the decision to publish the results. 

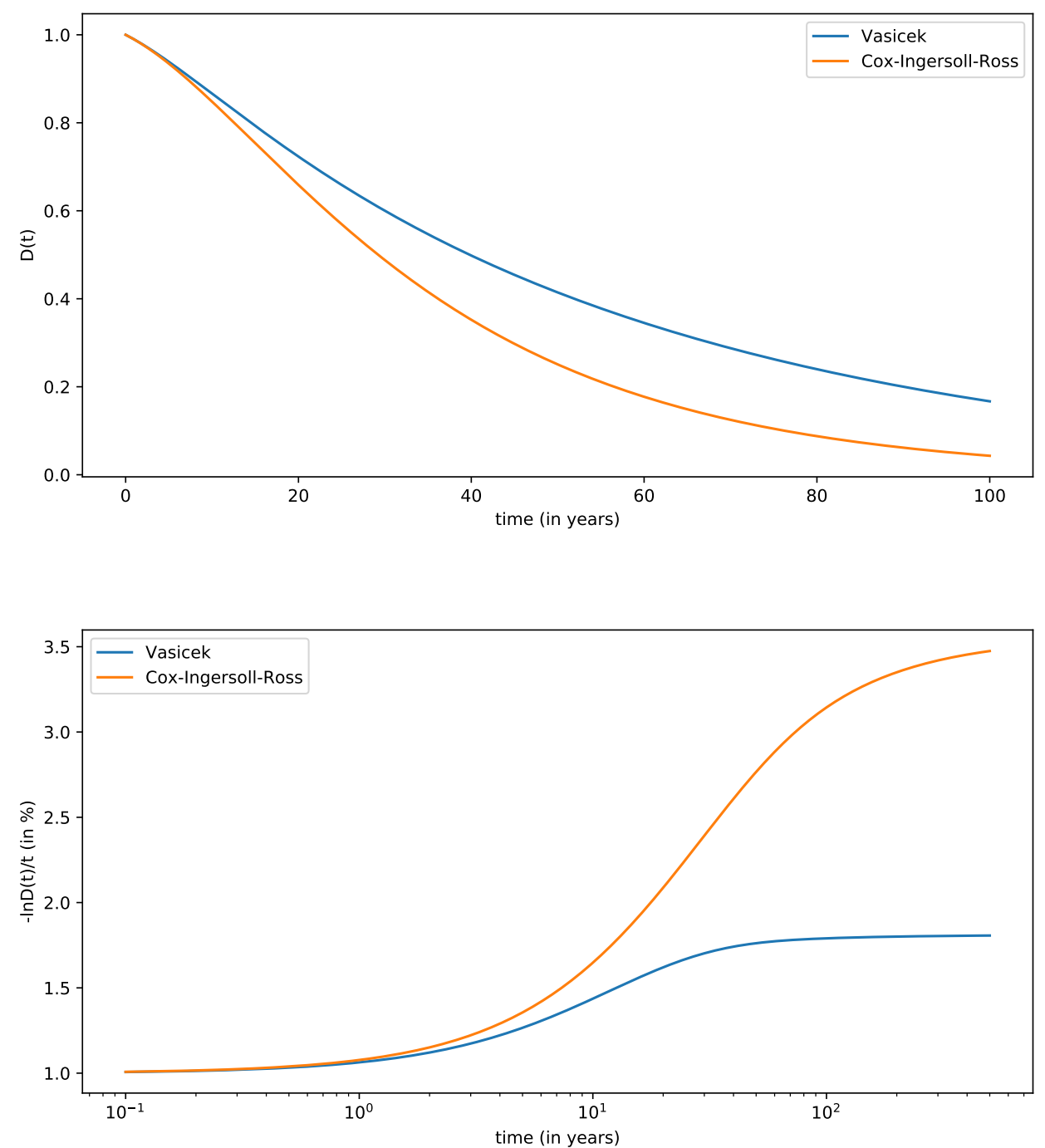

Figure 1. The Vasicek and Cox-Ingersoll-Ross discount functions. The parameters used are those corresponding to the United States and are provided by Table 5 of Ref. [28] (see Section 5). In the top figure we plot the discount function $D(t)$ while in the bottom figure we plot the log ratio $-\ln D(t) / t$. In the top figure we observe the asymptotic exponential decay of the discount after more than a hundred years, while in the bottom figure it is clearly seen the existence of a long-run discount rate for the Vasicek model (cf. Eq. (82)). The initial rate $r_{0}$ is arbitrarily taken to be $1 \%$. In both models we assume no market price of risk $q(r)=0$ (Local Expectation Hypothesis). 


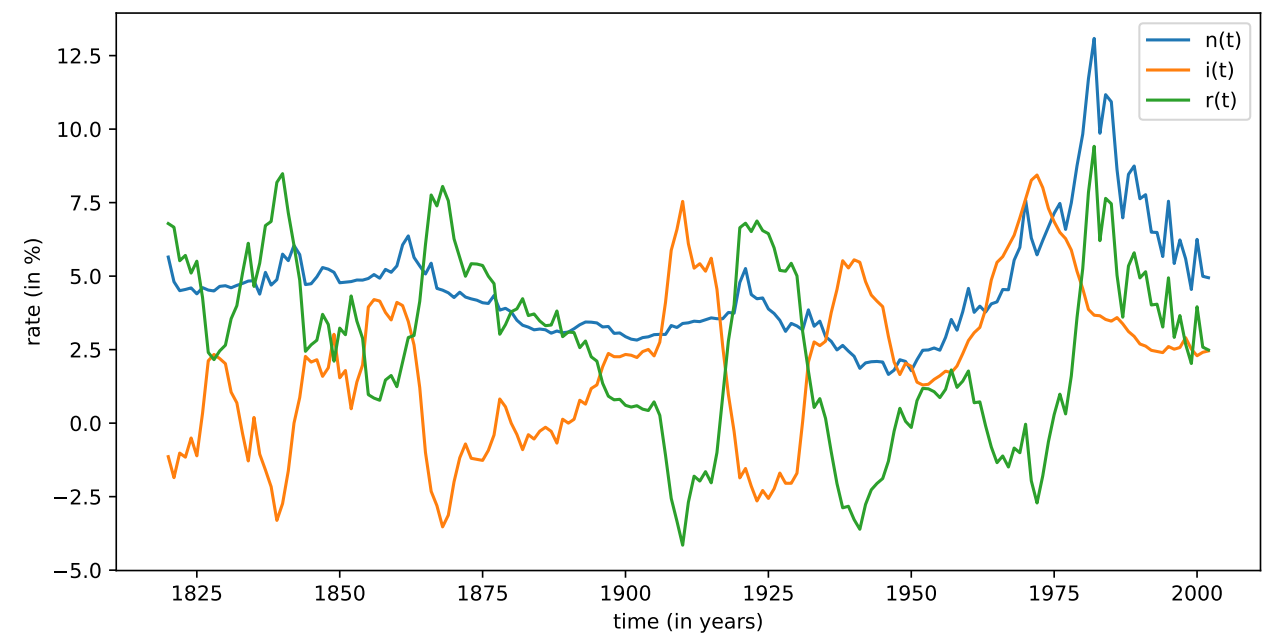

Figure 2. Real interest rates display large fluctuations and negative rates are not uncommon. We show nominal $n(t)$, inflation $i(t)$, and real interest rates $r(t)$ for the United States (USA). 


\section{References}

1. R. N. Mantegna and H. E. Stanley, Introduction to econophysics: correlations and complexity in finance (Cambridge University Press, Cambridge, 1999)

2. J.-P. Boucahaud and M. Potters, Theory of financial risk and derivative pricing: from statistical mechanics to risk management (Cambridge University Press, Cambridge, 2011)

3. J. P. Bouchaud, “Econophysics: still fringe after 30 years?", Europhysics News 50 24-27 (2019)

4. L. Bachelier, “Théorie de la spéculation", Ann. Sci. École Norm. Sup. 17, 21-86 (1900). Reprinted in P. H. Cootner (Ed.), The random character of stock market prices (MIT Press, Cambridge, MA, 1964)

5. M. F. M. Osborne, “Brownian motion in stock markets", Operations Research 7, 145-173 (1959). Reprinted in P. H. Cootner (Ed.), The random character of stock market prices (MIT Press, Cambridge, MA, 1964)

6. R. Cont, "Empirical properties of asset returns: stylized facts and statistical issues", Quant. Finance 1, 223-236 (2001)

7. B. Mandelbrot, "The variation of certain speculative prices", Journal of Business 35, 394-419 (1963)

8. E. Fama, "Mandelbrot and the stable Paretian hypothesis", Journal of Business 35, 420-429 (1963)

9. E. Stein and J. Stein, "Stock price distributions with stochastic volatility: an analytic approach", Rev. Financ. Stud. 4, 727-752 ( 1991)

10. J.-P. Fouque, G. Papanicolau, and K. R. Sircar, Derivatives in financial markets with stochastic volatility (Cambdrige University Press, Cambridge, 2000)

11. J. Masoliver and J. Perelló, "Multiple time scales and the exponential Ornstein-Uhlenbeck stochastic volatility model", Quant. Finance 6, 423-433 (2006).

12. P. Samuelson, "A note on measurement of utility", Rev Econ Stud 4, 155-161 (1937).

13. D. Brigo and F. Mercurio, Interest Rate Models - Theory and Practice (Springer-Verlag, Berlin, 2006).

14. K. J. Arrow, M. L. Cropper, C. Gollier, B. Groom, B., G. M. Heal, R. G. Newell, W. D. Nordhaus, R. S. Pindyck, W. A. Pizer, P. R. Portney, T. Sterner, R. S. J. Tol, M. L. Weitzman, “Determining benefits and costs for future generations”, Science 341, 349-350 (2013)

15. N. Stern, The Economics of climate change: The Stern Review (Cambridge University Press, Cambridge, 2006)

16. W. D. Nordhaus, "The Stern Review on the economics of climate change", J Econ Literature 45, 687-702 (2007)

17. W. D. Nordhaus, "Critical assumptions in the Stern Review on Climate Change", Science 317, 201-202 (2007)

18. P. Dasgupta, Comments on the Stern Review's Economics of Climate Change (Cambridge University Press, Cambridge, 2006)

19. M. L. Weitzman, "A review on the Stern review on the economics of climate change". Journal of Economics Literature 45, 703-724 (2007)

20. W. D. Nordhaus, A Question of Balance (Yale University Press, New Haven, 2008)

21. N. Stern, "Ethics, equity and the economics of climate change. Paper 1", Science and philosophy. Economics and Philosophy 30, $397(2014)$

22. N. Stern, "Ethics, equity and the economics of climate change. Paper 2", Science and philosophy. Economics and Philosophy 30, $445(2014)$

23. m. a. Drupp, M. A., M. C. Freeman, B. Groom, and F. Nesje, “Discounting disentangled", American Economic Journal: Economic Policy 10, 109-134 (2018)

24. G. M. Heal and A. Millner, "Agreeing to disagree on climate policy”, PNAS 111, 3695-3698 (2014)

25. F. P. Ramsey, "A mathematical theory of saving", Economic Journal 38, 543-559 (1928).

26. J. D. Farmer, J. Geanakoplos, J. Masoliver, M. Montero and J. Perelló, “Discounting the distant future", University of Yale, Cowles Foundation Discussion Paper no. 1951 (2014). (Available at SSRN: http://ssrn.com/Abstract=1448811)

27. J. D. Farmer, J. Geanakplos and J. Masoliver and M. Montero and J. Perelló, "Value of the future: Discounting in random environments", Phy. Rev. E 91, 052816 (2015).

28. J. Perelló, M. Montero, J. Masoliver, J. D. Farmer and J. Geanakoplos, "Statistical analysis and stochastic interest rate modeling for valuing the future with implications in climate change mitigation", J. Stat. Mech. (2020) 0432110

29. J. D. Farmer, J. Geanakoplos, J. Masoliver, M. Montero, J. Perelló J and M. G. Richiardi, “Discounting the distant future: What do historical bond prices imply about the long term discount rate?", J. Math. Economics (to appear, 2021)

30. L. B. G. Andersen and V. V. Piterberg, Interest rate modeling, Vols. I - III (Atlantic Financial Press, 2010)

31. D. Duffie, "Credit risk modeling with affine processes", J. Banking and Finance 29, 2751-2802 (2005)

32. J. Masoliver, Random processes, first-passage and escape (World Scientific, Singapore, 2018).

33. C. W. Gardiner, Handbook of stochastic methods (Springer-Verlag, Berlin,1986).

34. J. Masoliver, M. Montero and J. Perelló, "Valuing the future under random structural conditions: Non-stationary models for discounting" (in preparation, 2021).

35. C. Cox, J. E. Ingersoll, S. A. Ross, “A re-examination of the traditional hypothesis about the term structure of interest rates", J. Finance 35, 769-799 (1981).

36. C. Gilles, S. F. Leroy, "A note on the local expectation hypothesis", J. Finance 41, 975-979 (1986).

37. O. Vasicek "An equilibrium characterization of the terms structure", J. Fin. Economics 5, 177-188 (1977).

38. M. Piazzesi, "Affine term structure models"; in Y. A. Sahala and M. P. Hansen (Eds.) The Handbook of Financial Econometrics pp.691-766 (Elsevier, Amsterdam, 2009). 
39. W. F. Sharpe, "Mutual fund performance", J. Business 39, 119-138 (1966).

40. J. Masoliver and J. Perelló, "First-passage and escape problems in the Feller process", Phys. Rev. E 86, 041116 (2012)

41. J. C. Cox, J. E. Ingersoll, and S. A. Ross, "A theory of the term structure of interest rates", Econometrica 53, 385 (1985)

42. W. Feller, "Two singular diffusion processes", Ann. Math.54, 173 (1951)

43. L. U. Dothan, "On the term structure of interest rates", J. Financial Economics 6, 59-69 (1978).

44. H. R. Pitt Tauberian Theorems (Oxford University Press, London, 1958).

45. R. A. Handelsman and J. S. Lew, "Asymptotic expansion of Laplace convolutions for large argument and tail densities for certain sums of random variables", SIAM J. Math. Anal. 5, 425 (1974).

46. J. D. Farmer and J. Geanakoplos, "Hyperbolic discounting is rational: Valuing the far future with uncertain discount rates", Cowles Foundation Discussion Paper No. 1719, 2009. (Available at SSRN: http:/ /ssrn.com/abstract=1448811).

47. J. Geanakoplos, W. Sudderth, and O. Zeitouini, “Asymptotic behavior of stochastic discount rates”, Ind. J. Stat. 76 A, 150 (2014).

48. R. Newell, N. Pizer, "Discounting the distant future: How much do uncertain rates increase valuations?", J Environ Econ and Management 46, 52-71 (2003)

49. C. Gollier, P Koundouri, T. Pantelidis, "Declining discount rates: Economic justifications and implications for long-run policy", Economic Policy 23, 757-795 (2008)

50. S. Giglio, M. Maggiori, J. Stroebel, “Very long-run discount rates”, Quarterly Journal of Economics 130, 1-53 (2015)

51. S. Giglio, M. Maggiori, J. Stroebel, “No-bubble conditions: Model-free test in housing markets”, Econometrica 84, 1047-1091 (2016)

52. M. L. Weitzman, "Why the far-distant future should be discounted at its lowest possible rate", J. Environment Econ and Management, 36 201-208 (1998)

53. B. Groom, P. Koundouri, E. Panopoulou, T. Pantelidis, “Discounting distant future: How much selection affect the certainty equivalent rate", J. Appl. Econometrics 22, 641-656 (2007)

54. C. Hepburn, P. Koundouri, E. Panopoulou, T. Pantelidis, "Social discounting under uncertainty: a cross-country comparison", J. Environ. Econ. and Management 57, 140-150 ( 2007)

55. M. C. Freeman, B. Groom, E. Panopoulou, T. Pantelidis, “Declining discount rates and the Fisher Effect: Inflated past, discounted future?", J. Environ. Econ. and Management 73, 32-49 (2015)

56. Tang C Y, Chen S X, Parameter estimation and bias correction for diffusion processes 2009 Journal of Econometrics 149(1) 65-81 